\title{
SPECTRAL BOUNDS USING HIGHER-ORDER NUMERICAL RANGES
}

\author{
E. B. DAVIES
}

\begin{abstract}
This paper describes how to obtain bounds on the spectrum of a non-self-adjoint operator by means of what are referred to here as 'its higher-order numerical ranges'. Proofs of some of their basic properties are given, as well as an explanation of how to compute them. Finally, they are used to obtain new spectral insights into the non-self-adjoint Anderson model in one and two space dimensions.
\end{abstract}

\section{Introduction}

Let $A$ be a bounded non-self-adjoint (NSA) operator on a Hilbert space $\mathscr{H}$. We have

$$
\operatorname{Spec}(A) \subseteq \operatorname{Num}(A) \subseteq\{z:|z| \leqslant\|A\|\},
$$

where $\operatorname{Num}(A)$ is the closure of the numerical range of $A$. Although $\operatorname{Num}(A)$ is easy to compute, it is always a convex set, and so does not usually provide much information about the spectrum. One of the goals of this paper is to obtain tighter bounds on the spectrum of $A$ by using what we call 'higher-order numerical ranges', defined below. The optimal result of this type is Theorem 1.3, but we concentrate mainly on the properties of the quadratic numerical range, which can be studied theoretically as well as numerically. In Theorem 1.6, we prove that $\operatorname{Spec}(A)$ is contained inside a family of hyperbolic regions, which are explicitly determined in the examples that we study later in the paper. This provides much sharper information than is available by using (1).

This paper originated with the study by Martínez and the author of the spectrum of the non-self-adjoint (NSA) Anderson model. The result is two papers. In this one, we prove some general theorems about the quadratic and higher-order numerical ranges. In [22], Martínez proves surprisingly detailed results for the NSA Anderson model in one space dimension, using quadratic numerical ranges. For some values of the parameters, she is able to determine the spectra of the relevant NSA Anderson models completely.

The main definitions and general properties of the polynomial numerical ranges are given in this section. The remaining sections of the paper fall into three independent groups, and illustrate different aspects, or applications, of the ideas. In Section 2, we describe the relationship between polynomial numerical ranges and pseudospectra, as described in [30]. Section 3 obtains uniform bounds on the spectra of a structured family of (large, non-selfadjoint) perturbations of a given self-adjoint operator; applications to Schrödinger operators with complex-valued potentials are given.

Sections 4, 5 and 6 all consider the non-self-adjoint (NSA) Anderson model. In Section 4, we set the scene and provide some introductory results. Section 5 is devoted to adapting the use of quadratic numerical ranges as found in [22], from one to higher dimensions. Finally, 
Section 6 provides a series of numerical examples that together illustrate the strength of the technique. These concentrate on cases, previously neglected, in which there are high spatial correlations between the values of the potential at neighbouring points.

We now turn to the concepts that form the subject matter of the paper. If $p$ is any polynomial, then

$$
p(\operatorname{Spec}(A))=\operatorname{Spec}(p(A)) \subseteq \operatorname{Num}(p(A)) .
$$

Therefore,

$$
\operatorname{Spec}(A) \subseteq \operatorname{Num}(p, A):=\{z: p(z) \in \operatorname{Num}(p(A))\} .
$$

The above definitions and certain of the results below can be extended to suitable analytic functions. We put

$$
\operatorname{Num}_{n}(A):=\bigcap_{\operatorname{deg}(p) \leqslant n} \operatorname{Num}(p, A)
$$

for any positive integer $n$, and

$$
\operatorname{Num}_{\infty}(A):=\bigcap_{n} \operatorname{Num}_{n}(A)=\bigcap_{p} \operatorname{Num}(p, A) .
$$

If $\alpha, \beta \in \mathbb{C}$ and $\alpha \neq 0$, then

$$
\operatorname{Num}(\alpha p+\beta, A)=\operatorname{Num}(p, A),
$$

from which it follows that in the definition of $\operatorname{Num}_{n}(A)$ one need only consider polynomials with leading coefficient 1 and constant coefficient 0 .

The polynomial numerical ranges just defined are obviously contained in the polynomial numerical hulls of Nevanlinna $[\mathbf{1 2}, \mathbf{1 7}, \mathbf{2 4}$, which we define by

$$
\operatorname{Hull}(p, A):=\{z:|p(z)| \leqslant\|p(A)\|\}
$$

and

$$
\operatorname{Hull}_{n}(A)=\bigcap_{\operatorname{deg}(p) \leqslant n} \operatorname{Hull}(p, A)
$$

The fact that

$$
\operatorname{Num}_{n}(A)=\operatorname{Hull}_{n}(A)
$$

for all $n$ and all bounded $A$ is far from obvious, and was proved very recently by Burke and Greenbaum [6]. Note that $\operatorname{Num}_{n}(A)$ makes sense if $A$ is unbounded, but $\operatorname{Hull}_{n}(A)$ does not.

We mention that although we call $\operatorname{Num}_{2}(A)$ 'the quadratic numerical range of $A$ ', it has no obvious connection with the concept of the same name in $[\mathbf{2 0 , 2 1}$. They define a set $W^{2}(\mathcal{A}) \subseteq \mathbb{C}$, but only for a $(2 \times 2)$-block operator matrix $\mathcal{A}$. Their set can have only one or two components, whereas $\operatorname{Num}_{2}(A)$ may have many: for example, if $A=A^{*}$, then $\operatorname{Num}_{2}(A)=\operatorname{Spec}(A)$.

We describe below how to use the following lemma to determine $\operatorname{Num}(p, A)$ numerically.

LEMMA 1.1. Let $K \subseteq \mathbb{R}^{N}$ be a compact set with boundary $\partial K$ and non-empty interior $\operatorname{Int}(K)$, and let $p: \mathbb{R}^{N} \longrightarrow \mathbb{R}^{N}$ be a continuous function such that $|p(x)| \rightarrow \infty$ as $|x| \rightarrow \infty$. Let $S=p^{-1}(K)$ and $B=p^{-1}(\partial K)$. Then $S$ is the union of $B$ and one or more components of $\mathbb{R}^{N} \backslash B$. 
Proof. It is immediate from the assumptions that $S$ is a compact set, and that $B$ is a proper subset of $S$. If $U$ is a component of $\mathbb{R}^{N} \backslash B$, then $U$ is covered by the two disjoint open sets $p^{-1}(\operatorname{Int}(K))$ and $p^{-1}\left(\mathbb{R}^{N} \backslash K\right)$, so one of these must be empty.

In the above lemma, let $N=2$, and let $p$ be a polynomial in the complex variable $z$. Let $K=\operatorname{Num}(p(A))$ for some bounded operator $A$. Then $K$ is convex and $\partial K$ is a simple closed curve that may be parametrized by the angles of the tangent lines. By solving $p(z)=w$ for each $w \in \partial K$, we see that $B$ comprises one or more closed curves, which may cross each other or themselves. These curves have the same degree of regularity as $\partial K$ away from the critical points of $p$. To determine whether any particular bounded component $U$ of $\mathbb{C} \backslash B$ is contained in $S$, we need only test whether $p(z) \in K$ for a single $z \in U$.

In the context of this example, the following lemma completes the determination of $\operatorname{Num}(p, A)$.

Lemma 1.2. If we assign a component $U$ of $\mathbb{C} \backslash B$ the parity 1 if and only if $U \subseteq S$, then neighbouring components (that is, components whose boundaries contain some common arc in B) have opposite parity.

Proof. One needs only to consider the behaviour of $p$ in the neighbourhood of any point $a \in B$ that is not one of the finite number of critical points of $p$.

We next consider how much information about $\operatorname{Spec}(A)$ can in principle be obtained by determining $\operatorname{Num}(p, A)$ for various choices of $p$. The equality of the second and third terms of (5) is due to Nevanlinna [24], while the equivalence of the first and third terms is due to the author. The observation that the two results are equivalent follows immediately from the recent paper of Burke and Greenbaum [6], by the use of (4).

Theorem 1.3 (Nevanlinna, Davies, Burke, Greenbaum). Let A be a bounded linear operator on $\mathcal{H}$, and let $\widehat{\operatorname{Spec}}(A)$ denote the complement of the unbounded component of $\mathbb{C} \backslash \operatorname{Spec}(A)$; that is, $\operatorname{Spec}(A)$ together with all regions enclosed by this set. Then

$$
\operatorname{Num}_{\infty}(A)=\operatorname{Hull}_{\infty}(A)=\widehat{\operatorname{Spec}}(A) .
$$

Proof. Our proof relies on published sources, and can be adapted to certain unbounded operators, which no proof based on (4) could. It follows directly from the definitions that

$$
\operatorname{Num}_{\infty}(A) \subseteq \operatorname{Hull}_{\infty}(A) .
$$

If $a \notin \operatorname{Num}_{\infty}(A)$, then there exists a polynomial $p$ such that $p(a) \notin \operatorname{Num}(p(A))$. Since $\operatorname{Num}(p(A))$ is closed and convex, there exists a real-linear functional $\phi: \mathbb{C} \longrightarrow \mathbb{R}$ such that $\phi(p(a))<0$ and

$$
\phi(\operatorname{Spec}(p(A))) \subseteq \phi(\operatorname{Num}(p(A)) \subseteq[0, \infty) .
$$

Since $\operatorname{Spec}(p(A))=p(\operatorname{Spec}(A))$, the harmonic function $\psi: \mathbb{C} \longrightarrow \mathbb{R}$ defined by $\psi(z)=$ $\phi(p(z))$ satisfies $\psi(a)<0$, and

$$
\psi(\operatorname{Spec}(A)) \subseteq[0, \infty) .
$$

Using the maximum principle, we see not only that $a \notin \operatorname{Spec}(A)$, but also that $a$ does not lie in any bounded component of the complement of the spectrum. Therefore $a \notin \widehat{\operatorname{Spec}}(A)$. This implies that

$$
\widehat{\operatorname{Spec}}(A) \subseteq \operatorname{Num}_{\infty}(A) .
$$


The proof is completed by combining (6), (7) and the second equality of (5), which was proved by Nevanlinna in [24, Theorem 2.10.3].

We now specialize to polynomials of degree 2.

LEMMA 1.4. If $p_{c}(z)=(z-c)^{2}$, then either $\operatorname{Num}\left(p_{c}, A\right)$ is a star-shaped region with centre $c$, or there exists $\gamma \in \mathbb{R}$ and a closed simply connected set

$$
S \subseteq\{z: \gamma \leqslant \arg (z) \leqslant \gamma+\pi / 2\}
$$

such that

$$
\operatorname{Num}\left(p_{c}, A\right)=(c+S) \cup(c-S) .
$$

Proof. By replacing $A$ by $A-c I$, we are able to reduce to the case $c=0$. Let $K=$ $\operatorname{Num}\left(A^{2}\right)$, and consider first the case in which 0 lies in the interior of $K$. Since $K$ is convex, there exists a positive continuous periodic function $\rho(\theta)$ such that

$$
K=\left\{r \mathrm{e}^{i \theta}: 0 \leqslant r \leqslant \rho(\theta), 0 \leqslant \theta \leqslant 2 \pi\right\} .
$$

It follows that

$$
\operatorname{Num}\left(p_{0}, A\right)=\left\{r \mathrm{e}^{i \theta}: 0 \leqslant r \leqslant \rho(2 \theta)^{1 / 2}, 0 \leqslant \theta \leqslant 2 \pi\right\} .
$$

Therefore $\operatorname{Num}\left(p_{0}, A\right)$ is star-shaped, with centre at 0 .

Alternatively, suppose that 0 is not in the interior of $K$. Whether it lies outside $K$ or on the boundary of $K$, the convexity of $K$ implies that there exist $\alpha, \beta \in \mathbb{R}$ and continuous functions $\rho_{j}:[\alpha, \beta] \longrightarrow \mathbb{R}$ such that $\alpha<\beta \leqslant \alpha+\pi$ and $0 \leqslant \rho_{1}(\theta)<\rho_{2}(\theta)$ for all $\theta \in(\alpha, \beta)$ and

$$
K=\left\{r \mathrm{e}^{i \theta}: \theta \in[\alpha, \beta] \text { and } \rho_{1}(\theta) \leqslant r \leqslant \rho_{2}(\theta)\right\} .
$$

The second alternative of the lemma follows with

$$
S=\left\{r \mathrm{e}^{i \theta}: \theta \in[\alpha / 2, \beta / 2] \text { and } \rho_{1}(2 \theta)^{1 / 2} \leqslant r \leqslant \rho_{2}(2 \theta)^{1 / 2}\right\} .
$$

The following lemma shows that little new information about $\operatorname{Spec}(A)$ is obtained if $|c|$ is large. When we refer to the convergence of compact sets here and below, we mean with respect to the Hausdorff metric.

Lemma 1.5. If $|c|>4\|A\|$ and $p_{c}(z)=(z-c)^{2}$, then $\operatorname{Num}\left(p_{c}, A\right)$ is the union of two disjoint parts $A_{c}$ and $B_{c}$. The set $A_{c}$ is contained in $\{z:|z| \leqslant 3\|A\|\}$, and converges to $\operatorname{Num}(A)$ as $|c| \rightarrow \infty$. The set $B_{c}$ is contained in $\{z:|z| \geqslant 2|c|-3\|A\|\}$, and moves away to infinity as $|c| \rightarrow \infty$.

Proof. We put $q_{c}(z)=z-z^{2} /(2 c)$ and use (3) to show that $\operatorname{Num}\left(p_{c}, A\right)=\operatorname{Num}\left(q_{c}, A\right)$. Since

$$
\left|\left\langle q_{c}(A) f, f\right\rangle-\langle A f, f\rangle\right|<\frac{\|A\|^{2}}{|2 c|}
$$

provided that $\|f\|=1$, we see that $\operatorname{Num}\left(q_{c}(A)\right)$ converges to $\operatorname{Num}(A)$ as $|c| \rightarrow \infty$. We have to find bounds on all solutions of $q_{c}(z)=w$ where $w \in \operatorname{Num}\left(q_{c}(A)\right)$ satisfies $|w| \leqslant\|A\|+\|A\|^{2} /|2 c|$.

The quadratic equation possesses two solutions:

$$
z_{ \pm}=c \pm c \sqrt{1+u}
$$


where $u=-2 w / c$ satisfies $|u| \leqslant 1$ under the stated conditions. Using the inequality

$$
|\sqrt{1+u}-1| \leqslant|u|,
$$

valid for such $u$, we see that

$$
\left|z_{-}\right| \leqslant|c u|=2|w|=2\|A\|(1+\|A\| /|2 c|) \leqslant 3\|A\| .
$$

A similar argument shows that

$$
\left|z_{+}\right| \geqslant 2|c|-3\|A\| \geqslant 5\|A\| .
$$

The following properties of $\operatorname{Num}_{2}(A)$ can be deduced from its definition, and will be used below.

We define a hyperbolic region in the plane to be the connected region outside a rightangled hyperbola, or the two-component region inside such a hyperbola.

THEOREM 1.6. The complement of $\operatorname{Num}_{2}(A)$ is the union of a family of hyperbolic regions and half-planes. If $z \notin \operatorname{Num}_{2}(A)$, then

$$
S_{z}=\left\{\theta \in[0,2 \pi]_{\mathrm{per}}:\left(z+\mathrm{e}^{i \theta}[0, \infty)\right) \cap \operatorname{Spec}(A)=\emptyset\right\}
$$

contains an interval of length at least $\pi / 2$, or two disjoint intervals of combined length at least $\pi / 2$.

Proof. We have

$$
\mathbb{C} \backslash \operatorname{Num}_{2}(A)=\bigcup_{\operatorname{deg}(p) \leqslant 2}\{z: p(z) \notin \operatorname{Num}(p(A))\} .
$$

Since $\operatorname{Num}(p(A))$ is convex,

$$
\{z: p(z) \notin \operatorname{Num}(p(A))\}=\bigcup_{H \in \mathcal{F}}\{z: p(z) \notin H\},
$$

where $\mathcal{F}$ is a certain family of open half-planes $H$. Since each $p$ is of degree at most 2, each set $\{z: p(z) \notin H\}$ is a hyperbolic region or a half-plane. If $z \notin \operatorname{Num}_{2}(A)$, then there exists a hyperbolic region $U$ such that $z \in U$ and $U \cap \operatorname{Spec}(A)=\emptyset$. The final statement of the theorem now follows, by elementary geometry.

\section{Connection with pseudospectra}

The spectrum of an NSA operator or a large NSA matrix may be very unstable under small perturbations. This being so, any numerical method for determining or bounding the spectrum must have limited accuracy in regions of the complex plane where there is high spectral instability. In this section, we demonstrate this effect for the higher-order numerical ranges by finding their relationship with the pseudospectra of the operator concerned. The theory of pseudospectra is by now a well-established part of spectral analysis; we summarize the basic definitions, but cite $[3,4,29,30]$ for proofs and further information.

If $A$ is a bounded operator on $\mathscr{H}$, we define its pseudospectra as the family of sets

$$
\operatorname{Spec}_{\varepsilon}(A)=\operatorname{Spec}(A) \cup\left\{z:\left\|(z I-A)^{-1}\right\|>\varepsilon^{-1}\right\}
$$

parametrized by $\varepsilon>0$. 
One may also view pseudospectra as describing collective properties of a family of perturbations of $A$, because of the formula

$$
\operatorname{Spec}_{\varepsilon}(A)=\bigcup_{\|X\|<\varepsilon} \operatorname{Spec}(A+X) .
$$

If $N_{\varepsilon}(B)$ denotes the $\varepsilon$-neighbourhood of a set $B \subseteq \mathbb{C}$, then the formulae

$$
N_{\varepsilon}(\operatorname{Spec}(A)) \subseteq \operatorname{Spec}_{\varepsilon}(A) \subseteq N_{\varepsilon}(\operatorname{Num}(A))
$$

provide some weak information about the location of the pseudospectra. The following theorem provides a sharper result, using the polynomial numerical ranges. For the case $p(z)=z^{2}+c z$, the constant $k$ of the following theorem is $2\|A\|+|c|+1$.

THEOREM 2.1. If $A$ is a bounded operator, $p$ is a polynomial and $0<\varepsilon<1$, then there exists a constant $k$ depending on $p$ and $\|A\|$ such that

$$
\operatorname{Spec}_{\varepsilon}(A) \subseteq \operatorname{Num}_{k \varepsilon}(p, A),
$$

where

$$
\begin{aligned}
\operatorname{Num}_{\delta}(p, A) & =\{z: \operatorname{dist}\{p(z), \operatorname{Num}(p(A))\}<\delta\} \\
& =\left\{z: p(z) \in N_{\delta}(\operatorname{Num}(p(A)))\right\}
\end{aligned}
$$

Proof. If $p$ is of degree $n+1$, then

$$
p(z)-p(w)=\left(\sum_{r+s \leqslant n} a_{r, s} z^{r} w^{s}\right)(z-w) .
$$

If $z \in \operatorname{Spec}(A)$, then (10) is immediate. If $z \in \operatorname{Spec}_{\varepsilon}(A) \backslash \operatorname{Spec}(A)$, then there exists $f$ such that $\|f\|=1$ and $\|A f-z f\|<\varepsilon$. It follows that

$$
\begin{aligned}
\|p(z) f-p(A) f\| & <\sum_{r+s \leqslant n}\left|a_{r, s} z\right|^{r}\|A\|^{s} \varepsilon \\
& \leqslant k \varepsilon
\end{aligned}
$$

where

$$
k=\sum_{r+s \leqslant n}\left|a_{r, s}\right|(\|A\|+1)^{r}\|A\|^{s} .
$$

Therefore,

$$
|p(z)-\langle A f, f\rangle|<k \varepsilon .
$$

This implies that inclusion (10) holds.

The set $\operatorname{Num}_{\delta}(p, A)$ may be computed as follows. One first determines the boundary of $\operatorname{Num}(p(A))$, and then expands this outwards by the distance $\delta$ to get the boundary of the compact convex set $K=N_{\delta}(\operatorname{Num}(p(A)))$. One finally applies Lemmas 1.1 and 1.2 as in the determination of $\operatorname{Num}(p, A)$.

\section{Relatively bounded perturbations}

The main result of this section is Theorem 3.5. It enables one to obtain bounds on the location of the spectrum of a very large NSA matrix by computing the smallest eigenvalue 
of each of a small number of associated self-adjoint operators. Moreover, the same bounds apply to a class of matrices related to the chosen matrix in a specified manner. The bounds, or enclosures, of the spectrum are substantially better than those obtainable from the (firstorder) numerical range.

Let $A=H+B$, where $H$ is self-adjoint and $B$ is a relatively bounded perturbation with relative bound less than 1 , so that $A$ is closed with the same domain as $H$. We are interested in obtaining bounds on the spectrum of $A$ that apply simultaneously to all operators of the form $H+C B$ where $\|C\| \leqslant 1$. We identify a set $S \subseteq \mathbb{C}$ such that

$$
S=\bigcup_{\|C\| \leqslant 1} \operatorname{Spec}(H+C B),
$$

subject to a certain compactness condition. In contrast with (9), one observes that the perturbations in (11) have a very special structure imposed on them, but also that they are not required to be small.

Results such as the above are relevant in two situations. If one does not know the operator $B$ exactly, one may wish to discover how much can be said about the location of the spectrum of $A$. Alternatively, one may be interested in a randomly generated family of perturbations, and this family might be of the form (11). Lemma 3.1 is valid in a Banach space context.

Lemma 3.1. If we define $S=\{z: F(z) \geqslant 1\}$, where $F(z)=\left\|B(z I-H)^{-1}\right\|$ if $z \notin \operatorname{Spec}(H)$ and $F(z)=+\infty$ if $z \in \operatorname{Spec}(H)$, then

$$
\bigcup_{\|C\| \leqslant 1} \operatorname{Spec}(H+C B) \subseteq S .
$$

Proof. This relies upon the well-known formula

$$
(z I-H-C B)^{-1}=(z I-H)^{-1}\left(1-C B(z I-H)^{-1}\right)^{-1},
$$

which holds whenever $z \notin \operatorname{Spec}(H)$ and $\left\|C B(z I-H)^{-1}\right\|<1$.

The following lemma demonstrates that the pseudospectra of $A$ are relatively uninteresting outside the set $S$. Note that $H=H^{*}$ implies that

$$
\operatorname{Spec}_{\delta}(H)=\{z: \operatorname{dist}(z, \operatorname{Spec}(H))<\delta\}
$$

for all $\delta>0$.

Lemma 3.2. Given $0<\alpha<1$, put

$$
T=\{z: F(z) \leqslant \alpha\} .
$$

Then

$$
T \cap \operatorname{Spec}_{\varepsilon /(1+\alpha)}(H) \subseteq T \cap \operatorname{Spec}_{\varepsilon}(A) \subseteq T \cap \operatorname{Spec}_{\varepsilon /(1-\alpha)}(H)
$$

for all $\varepsilon>0$, provided that (8) holds.

Proof. This depends upon the bounds

$$
\left\|(z I-A)^{-1}\right\| \leqslant(1-\alpha)^{-1}\left\|(z I-H)^{-1}\right\|
$$

and

$$
\left\|(z I-H)^{-1}\right\| \leqslant(1+\alpha)\left\|(z I-A)^{-1}\right\|,
$$

which are valid for all $z \in T$ by virtue of (12). 
A systematic analysis of bounds on the spectra of non-self-adjoint Schrödinger operators is given in [11]. Two methods are described. One ultimately relies upon estimating $B(z I-H)^{-1}$ in the $L^{1}$ operator norm, and leads (among many much more general results) to the formula

$$
\operatorname{Spec}(-\Delta+V) \subseteq\left\{z:|z| \leqslant\|V\|_{1}^{2} / 4\right\}
$$

in one space dimension. See $[\mathbf{1}, \mathbf{2}, \mathbf{1 1}]$ for various derivations of this bound. The other method involves estimates of the Hilbert-Schmidt norm of $B(z I-H)^{-1}$. Both can be extended to $L^{2}\left(\mathbb{R}^{N}\right)$ in principle, but the results are not so useful.

The above methods depend on the existence of explicit expressions for the resolvent kernels of $-\Delta$, or they are restricted to one dimension. In this section we describe a different approach to bounding $\left\|B(z I-H)^{-1}\right\|$, which works in much greater generality. It is well adapted to computation, but it is not so easy to relate it to $L^{p}$-type norms of the potentials. We start by providing some insights into the structure of $S$.

LemMa 3.3. The function $F$ is locally Lipschitz continuous for $z \notin \operatorname{Spec}(H)$. For each $x \in \mathbb{R}$, the function $G: y \longrightarrow F(x+i y)$ is even and monotonic non-increasing as $|y|$ increases. If $B(H+i I)^{-1}$ is compact, then $G$ is strictly monotonic decreasing as $|y|$ increases and $\lim _{|y| \rightarrow \infty} G(y)=0$.

Proof. Let $K$ be a compact subset of $\mathbb{C} \backslash \operatorname{Spec}(H)$, and let $a \in K$. By virtue of the bound

$$
\left\|B(z I-H)^{-1}\right\| \leqslant\left\|B(a I-H)^{-1}\right\|\left\|(a I-H)(z I-H)^{-1}\right\|,
$$

there exists a constant $c$ such that

$$
\left\|B(z I-H)^{-1}\right\| \leqslant c
$$

for all $z \in K$. Suppose that the same estimate holds if we replace $B$ by $I$. Then, for any $z, w \in K$, we have

$$
\begin{aligned}
|F(z)-F(w)| & \leqslant\left\|B(z I-H)^{-1}-B(w I-H)^{-1}\right\| \\
& =\left\|B(z I-H)^{-1}(w-z)(w I-H)^{-1}\right\| \\
& \leqslant c^{2}|z-w| .
\end{aligned}
$$

The fact that $G$ is even depends upon the identity

$$
B(z I-H)^{-1}=B(\bar{z} I-H)^{-1} U,
$$

where $U$ is a unitary operator. If $0 \leqslant y \leqslant y^{\prime}, z=x+i y$ and $z^{\prime}=x+i y^{\prime}$, then $G(y) \geqslant G\left(y^{\prime}\right)$ follows from the identity

$$
B\left(z^{\prime} I-H\right)^{-1}=B(z I-H)^{-1} C
$$

where $C$ is a contraction.

Finally, suppose that $B(H+i I)^{-1}$ is compact. Then $G(y)^{2}=\lambda(y)$, where $\lambda(y)$ is the largest eigenvalue of the compact operator

$$
E_{y}=B\left((x I-H)^{2}+y^{2} I\right)^{-1} B^{*} .
$$

If $\phi_{y}$ are the corresponding normalized eigenvectors and $0<y^{\prime}<y$, then

$$
\lambda\left(y^{\prime}\right) \geqslant\left\langle E_{y^{\prime}} \phi_{y}, \phi_{y}\right\rangle>\left\langle E_{y} \phi_{y}, \phi_{y}\right\rangle=\lambda(y) .
$$

The proof is completed by observing that since $E_{y}$ are compact and decrease monotonically to 0 as $|y| \rightarrow \infty$, they also converge to 0 in norm. (This general theorem may be proved by treating the finite rank case first, and then using a density argument.) 


\section{Spectral bounds}

Under an extra condition, we can write Lemma 3.1 in a form that is sharper and relates more directly to $H$ and $B$. It is also better adapted to computation for very large, sparse matrices.

THeOREM 3.4. If $B(H+i I)^{-1}$ is compact, then

$$
S=\left\{x+i y: y^{2} \leqslant-\sigma(x)\right\},
$$

where $\sigma(x)$ is the smallest eigenvalue of

$$
K_{x}=(x I-H)^{2}-B^{*} B .
$$

If $(H+i I)^{-1}$ is also compact, then

$$
S=\bigcup_{\|C\| \leqslant 1} \operatorname{Spec}(H+C B) .
$$

Proof. We use standard variational methods for self-adjoint operators freely; see [8]. In particular, we define the self-adjoint operator $K_{x}$ to be that associated with the closed, semibounded, quadratic form

$$
Q_{x}(f)=\|(x I-H) f\|^{2}-\|B f\|^{2}
$$

whose form domain is $\operatorname{Dom}(H)$. We do not need to determine the operator domain of $K_{x}$. The key idea is that Lemma 3.3 implies that $\left\|B(z I-H)^{-1}\right\| \geqslant 1$ if and only if $\left\|B(w I-H)^{-1}\right\|>1$ for all $w=x+i u$ such that $0<|u|<|y|$. For each such $w$, the strict norm inequality holds if and only if there exists $f \neq 0$ such that

$$
\left\|B(w I-H)^{-1} f\right\|>\|f\| \text {. }
$$

This is equivalent to the existence of a non-zero $g \in \operatorname{Dom}(H)$ such that

$$
\|B g\|>\|(w I-H) g\|
$$

or

$$
\|B g\|^{2}>\|(x I-H) g\|^{2}+u^{2}\|g\|^{2}
$$

or

$$
0>Q_{x}(g)+u^{2}\|g\|^{2} .
$$

This holds for all $u$ such that $|u|<|y|$ if and only if

$$
0 \geqslant \sigma(x)+y^{2} \text {. }
$$

If $(H+i I)^{-1}$ is compact, then $K_{x}$ has discrete spectrum, and it possesses a normalized eigenfunction $\phi$ such that

$$
\left\langle\left\{(x I-H)^{2}-B^{*} B\right\} \phi, \phi\right\rangle=\sigma(x) .
$$

If $y \in S$, we deduce that

$$
\left\langle\left\{(x I-H)^{2}+y^{2} I\right\} \phi, \phi\right\rangle \leqslant\left\langle B^{*} B \phi, \phi\right\rangle
$$

or, equivalently,

$$
\|(z I-H) \phi\| \leqslant\|B \phi\| .
$$

Therefore there exists an operator $C$ with $\|C\| \leqslant 1$ such that

$$
(z I-H) \phi=C B \phi
$$

and $z$ is an eigenvalue of $H+C B$. The converse inclusion has already been proved in Lemma 3.1. 
We next obtain bounds on the spectrum of a different class of non-self-adjoint perturbations of $H$ by using second-order numerical ranges. Our results look rather similar, but Example 3.8 below shows that they are actually better.

We assume that $A=H+i W$, where $H$ and $W$ are both self-adjoint and $0 \leqslant W^{2} \leqslant V^{2}$ for some given self-adjoint operator $V$. We denote the set of all such $W$ by $\mathcal{V}$. As before, we assume that $V$ has relative bound less than 1 with respect to $H$. The bounds that we obtain depend on $H$ and $V$, but not on $W$, provided that it satisfies the above conditions. Our results can be applied to random Schrödinger operators $A=-\Delta+i W$ acting in $L^{2}(\Omega)$, in which $V$ is a given real-valued potential and $W$ is any real-valued potential such that $|W(x)| \leqslant|V(x)|$ for all $x \in \Omega$.

TheOREM 3.5. Given $c \in \mathbb{R}$, let $\sigma(c)$ denote the bottom of the spectrum of the operator $(H-c I)^{2}-V^{2}$. Then

$$
\bigcup_{W \in \mathcal{V}} \operatorname{Spec}(H+i W) \subseteq T \subseteq S,
$$

where

$$
T=\left\{x+i y: y^{2} \leqslant \tau(x)\right\}
$$

and

$$
\tau(x)=\inf _{c \in \mathbb{R}}\left\{(x-c)^{2}-\sigma(c)\right\} \leqslant-\sigma(x) \leqslant\|V\|^{2} .
$$

If $\tau(x)<0$ (possibly $\tau(x)=-\infty$ ), then

$$
\operatorname{Spec}(H+i W) \cap(x+i \mathbb{R})=\emptyset
$$

for all $W \in \mathcal{V}$.

Proof. If $x+i y \in \operatorname{Spec}(H+i W)$, then

$$
\begin{aligned}
\{(x-c)+i y\}^{2} & \in \operatorname{Spec}\left(\{(H-c I)+i W\}^{2}\right) \\
& \subseteq \operatorname{Num}\left(\{(H-c I)+i W\}^{2}\right) \\
& \subseteq\{w: \operatorname{Re}(w) \geqslant \sigma(c)\}
\end{aligned}
$$

by virtue of the quadratic form inequalities

$$
(H-c I)^{2}-W^{2} \geqslant(H-c I)^{2}-V^{2} \geqslant \sigma(c) I .
$$

Therefore $(x-c)^{2}-y^{2} \geqslant \sigma(c)$, or equivalently

$$
y^{2} \leqslant(x-c)^{2}-\sigma(c) .
$$

The proof of the first half of (13) is completed by taking the infimum over all $c \in \mathbb{R}$. The second half of (13) follows by putting $c=x$, and implies that $T \subseteq S$.

The following shows that the computation of $\tau(x)$ is not totally straightforward, even when the operators involved are all bounded. Let

$$
-\infty<s_{-}=\min (\operatorname{Spec}(H)) \leqslant \max (\operatorname{Spec}(H))=s_{+}<\infty,
$$

and let $V$ be bounded. Then

$$
\operatorname{Spec}(H+i W) \subseteq \operatorname{Num}(H+i W) \subseteq\left\{x+i y: s_{-} \leqslant x \leqslant s_{+} \text {and }|y| \leqslant\|V\|\right\},
$$

so it is not necessary to determine $\tau(x)$ for $x>s_{+}$or $x<s_{-}$. 
LEMMA 3.6. If $s_{-}<x<s_{+}$, then

$$
\tau(x)=\min _{c \in \mathbb{R}} \mu(x, c),
$$

where

$$
\mu(x, c)=\max \left(\operatorname{Spec}\left(x^{2} I-H^{2}+V^{2}+2(H-x I) c\right)\right)
$$

is a convex, Lipschitz continuous function of $c$ that diverges to $+\infty$ as $c \rightarrow \pm \infty$.

Proof. The identity (14) is elementary, apart from the question of whether it should have a min or an inf. Standard variational methods show that

$$
\left|\mu(x, c)-\mu\left(x, c^{\prime}\right)\right| \leqslant 2\|H-x I\|\left|c-c^{\prime}\right|
$$

for all $c, c^{\prime} \in \mathbb{R}$. Calculating to leading order only, variational methods also yield

$$
\begin{array}{ll}
\mu(x, c) \sim\left(s_{+}-x\right) c \rightarrow+\infty & \text { as } c \rightarrow+\infty \\
\mu(x, c) \sim\left(s_{-}-x\right) c \rightarrow+\infty & \text { as } c \rightarrow-\infty .
\end{array}
$$

These establish that $c \rightarrow \mu(x, c)$ has a minimum value. Indeed, since the operator in (15) depends linearly on $c$, the function $\mu(x, c)$ is a convex function of $c$, by a variational argument.

The problem mentioned is that the minimizing value of $c$ may become steadily larger as $x \rightarrow s_{-}$or $x \rightarrow s_{+}$. This leads to a residual inaccuracy near these points. If one minimizes with respect to $c$ in a fixed interval (or, more realistically, in a finite set), one obtains a larger and non-optimal value of $\tau(x)$, but the statements of Theorem 3.5 are still valid.

EXAMPLE 3.7. If we put

$$
H=\left(\begin{array}{cc}
-1 & 0 \\
0 & 1
\end{array}\right) \quad \text { and } \quad V=\left(\begin{array}{cc}
1 & 1 \\
1 & 1
\end{array}\right)
$$

then $s_{-}=-1, s_{+}=1$ and $\|V\|^{2}=2$. A direct calculation shows that

$$
\sigma(c)=c^{2}-1-2 \sqrt{c^{2}+1} .
$$

Therefore,

$$
\tau(1)=\inf _{c \in \mathbb{R}}\left\{2-2 c+2 \sqrt{c^{2}+1}\right\}=2 .
$$

The infimum is obtained by letting $c \rightarrow+\infty$. The same applies to $\tau(-1)$, but if $-1<x<1$ the corresponding minimum is achieved for a finite value of $c$.

EXAMPLE 3.8. We consider a discretization of the Airy operator defined on $L^{2}(-1,1)$. This operator arises as a special case of the Orr-Sommerfeld problem, and in the Torrey equation; see[25, 26, 27, 28]. Let $H f(x)=-h^{2} f^{\prime \prime}(x)$, subject to Dirichlet boundary conditions, and let $B=i V$, where $V$ is the operator of multiplication by the function $V(s)=s$ for all $s \in(-1,1)$. Redparth [26] has shown that if $V_{\delta}$ is a small perturbation of $V$, then the spectrum may have different limits, depending on the order in which one takes $h \rightarrow 0$ and $V_{\delta} \rightarrow V$ uniformly on $[0,1]$. These results motivate our example, but we consider the spectral properties of a particular large matrix $A_{N}$, not of the differential operator, let alone of the semi-classical limit. 


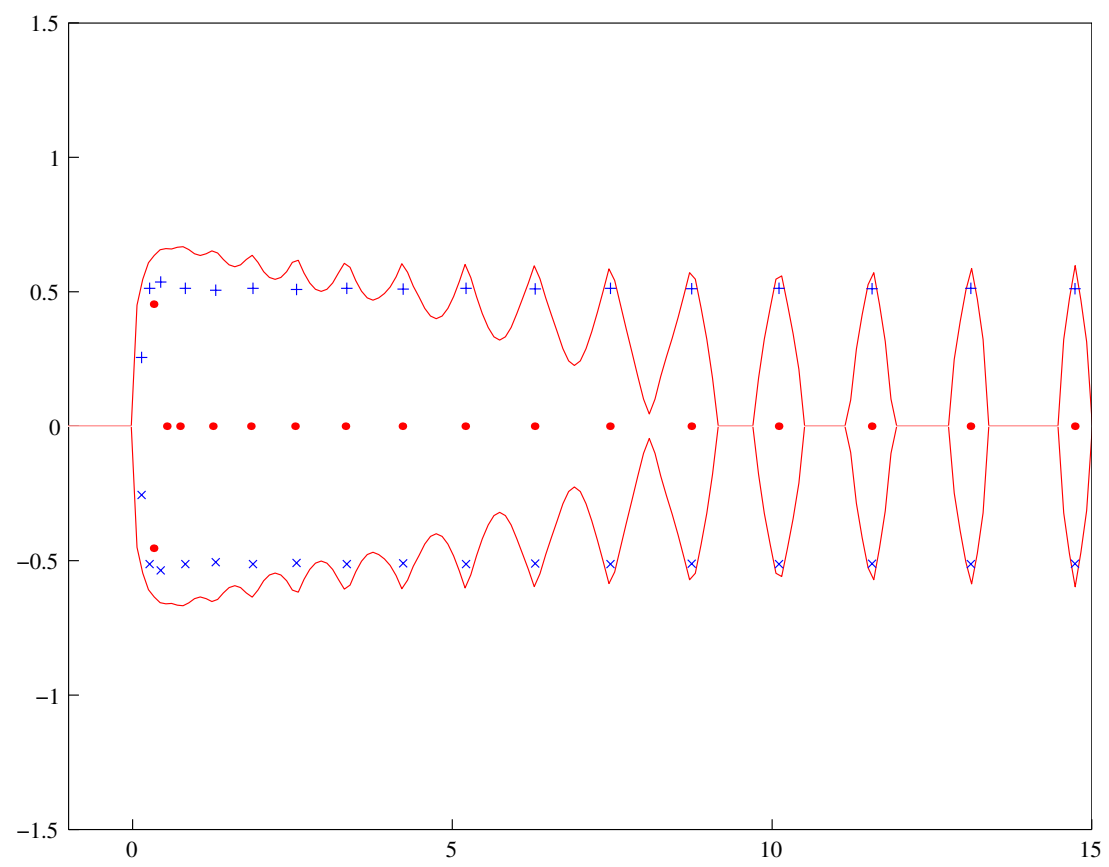

Figure 1: The set $T$ for $-1<x<15$.

We replace $(-1,1)$ by a finite set of $N=2 M+1$ points, parametrized by an integer $r$ such that $-M \leqslant r \leqslant M$. We replace the differential operator $H$ by the $N \times N$ matrix

$$
H_{r, s}= \begin{cases}-h^{2} M^{2} & \text { if }|r-s|=1, \\ 2 h^{2} M^{2} & \text { if } r=s, \\ 0 & \text { otherwise, }\end{cases}
$$

and we replace the operator $V$ by the diagonal matrix

$$
V_{r, s}= \begin{cases}r / M & \text { if } r=s \\ 0 & \text { otherwise. }\end{cases}
$$

We then apply the methods of this section to $A_{N}=H+i V$.

Figures 1 and 2 show the results of numerical computations for the case $M=40$, $h=0.15$. The curves are $y= \pm \tau(x)^{1 / 2}$, and bound the set $T$ defined in Theorem 3.5.

In Figure 1, the points marked '.' are the eigenvalues of $A_{N}$; the points marked ' + ' and ' $x$ ' are obtained by replacing $V$ by $|V|$ and $-|V|$, respectively. The potentials $\pm|V|$ are not small perturbations of $V$, so pseudospectral methods would not provide information about the spectra of the three operators (and all others in the class $\mathcal{V}$ ) simultaneously.

The isolated points in Figure 2 are the eigenvalues of 50 randomly selected operators (one eigenvalue for each operator), in which $V$ is replaced by matrices such that $V_{r, s}= \pm \delta_{r, s} r / M$ for every $r$. We chose the signs at successive values of $r$ to be strongly correlated. A plot expanded horizontally shows that the eigenvalues do not lie on a vertical line, but only close to one. 


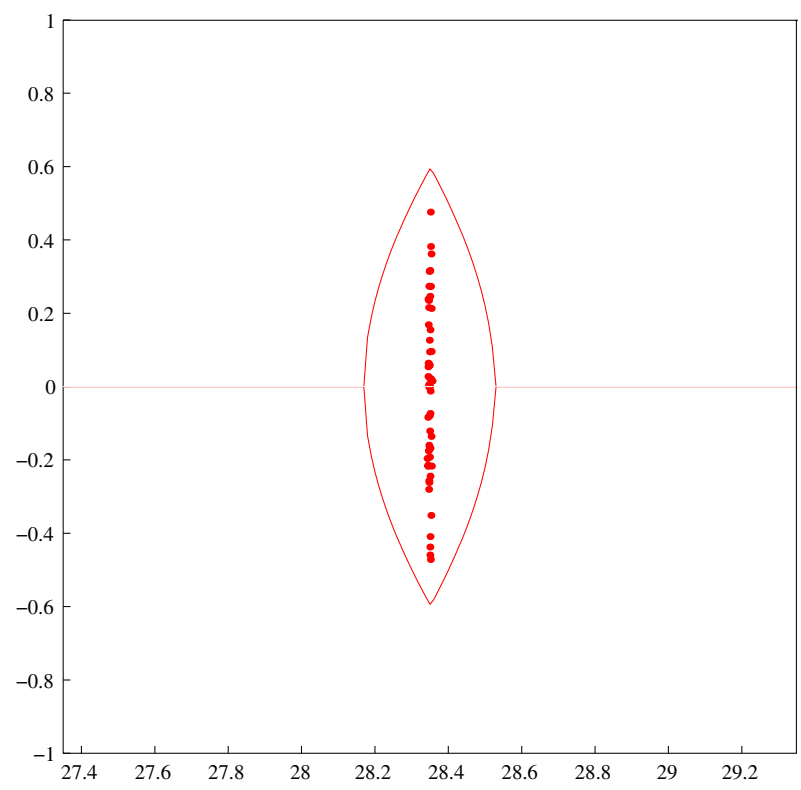

Figure 2: The set $T$ for $27.35<x<29.35$.

The following lemma proves a conjecture based on the above data about the asymptotics of $\operatorname{Re}(\operatorname{Spec}(A))$ as $x \rightarrow+\infty$.

Lemma 3.9. Let $A=H+i V$ act in $L^{2}(-1,1)$, where $H f(x)=-h^{2} f^{\prime \prime}(x)$ satisfies Dirichlet boundary conditions and $V$ is a bounded self-adjoint operator. Let $\lambda_{n}=\pi^{2} h^{2} n^{2} / 4$ denote the nth eigenvalue of $H$. If

$$
\pi^{2} h^{2}(2 n+1) \geqslant 8\|V\|,
$$

then

$$
\operatorname{Spec}(A) \cap\left\{z: \lambda_{n}+\delta_{n}<\operatorname{Re}(z)<\lambda_{n+1}-\delta_{n}\right\}=\emptyset
$$

where

as $n \rightarrow \infty$.

$$
\delta_{n}=\frac{8\|V\|^{2}}{\pi^{2} h^{2}(2 n+1)} \rightarrow 0
$$

Proof. We define $s$ and $t$ by

$$
\left\{\begin{aligned}
\lambda_{n} & =s-t, \\
\lambda_{n+1} & =s+t,
\end{aligned}\right.
$$

and we observe that (16) is equivalent to saying that $t \geqslant\|V\|$. We also observe that $z \in \operatorname{Spec}(A)$ implies that

$$
\begin{aligned}
(z-s)^{2} & \in \operatorname{Spec}\left((A-s I)^{2}\right) \\
& \subseteq \operatorname{Num}\left((A-s I)^{2}\right) \\
& \subseteq\{w: \operatorname{Re}(w) \geqslant \gamma\}
\end{aligned}
$$

for any $\gamma$ such that

$$
\gamma I \leqslant(H-s I)^{2}-V^{2}
$$


Taking $\gamma=t^{2}-\|V\|^{2}$, we obtain

$$
(x-s)^{2}-y^{2} \geqslant t^{2}-\|V\|^{2},
$$

and so

$$
|x-s| \geqslant \sqrt{t^{2}-\|V\|^{2}} \geqslant t\left(1-\|V\|^{2} / t^{2}\right) .
$$

Since $\delta_{n}=\|V\|^{2} / t$, this is equivalent to the statement of the lemma.

\section{The NSA Anderson model}

The NSA Anderson model was introduced by Hatano, Nelson and others [7, 18, 19, 23]; applications to non-hermitian quantum mechanics and to the growth of bacteria in an inhomogeneous environment were described. However, its purely mathematical properties turned out to be so interesting that it has recently been the subject of a number of mathematically rigorous papers. Goldsheid and Khoruzhenko considered the operator on a finite interval subject to periodic or quasi-periodic boundary conditions, and proved results about the spectrum of the operator in the limit as the length of the interval diverged $[14, \mathbf{1 5}, \mathbf{1 6}]$. They confirmed the earlier numerical studies indicating that the spectrum converges almost surely to a certain union of curves. They also obtained new results, rigorously deriving the asymptotic density of the eigenvalues on those curves.

On the other hand, the present author studied the same model, defined directly as a bounded linear operator acting on $l^{2}\left(\mathbb{Z}^{D}\right)$ for any $D$, and found that the spectrum consists of a bounded region in the complex plane; this is described in some detail when $D=1$ in $[9,10]$. Nevertheless, a number of spectral questions still needed to be clarified. Martínez has made substantial progress in resolving these [22], using the methods that we develop further in this paper.

One would expect to be able to investigate the infinite-volume limit using pseudospectral theory, and this has been done for a simple exactly soluble bidiagonal model [5, 13, 31]. Our goal here is to consider this issue from yet another point of view, and to demonstrate that the infinite-volume spectrum may be relevant to quite small finite systems. Whether or not this is true depends heavily on the dimension, and on the spatial correlations of the random potential; most numerical examples studied so far assume that the values of the potential at different points are statistically independent. This is a response to suggestions that the results of $[9, \mathbf{9 0}]$ concern extremely large deviations, which are irrelevant in finite physical systems; see, for example, [30]. The paper of Martínez gives further examples of the relevance of the infinite-volume spectrum for finite-volume systems in one dimension.

The assumptions in the earlier papers cited above vary considerably, and in this section we consider only $M \times M$ matrices $A$ such that

$$
A_{r, s}=\left\{\begin{aligned}
\alpha & \text { if } r=s-1 \text { or if } r=M, s=1, \\
\beta & \text { if } r=s+1 \text { or if } r=1, s=M, \\
v_{r} & \text { if } r=s, \\
0 & \text { otherwise, }
\end{aligned}\right.
$$

together with their infinite analogues. For finite matrices, we suppress the subscript $M$, but for infinite matrices we always include the subscript $\infty$. Here $\alpha$ and $\beta$ are distinct real constants, and the $v_{r}$ are distributed according to a stationary real-valued probability law. If one imposes Dirichlet boundary conditions (that is, $A_{M, 1}=A_{1, M}=0$ ), then the matrix is similar to a self-adjoint matrix, so its eigenvalues are all real. To obtain more interesting 
results, it is therefore standard to impose periodic or quasi-periodic boundary conditions, as in (17). The results obtained by Goldsheid and Khoruzhenko depend upon the condition that $\alpha \beta>0$, and upon the one-dimensionality of the model. On the other hand, when the conditions are satisfied, their spectral results are extremely detailed. The set $C$, to which the eigenvalues converge almost surely as the size of the matrix increases, depends upon the probability distribution used, but it is typically the union of a finite set of curves $[\mathbf{1 4}, \mathbf{1 5}, \mathbf{1 6}]$.

The papers of the author $[\mathbf{9 , 1 0}]$ work directly on $\mathbb{Z}$ (and can be extended to $\mathbb{Z}^{D}$ ). They assume that $A_{\infty}=H+V$ is a bounded operator on $l^{2}(\mathbb{Z})$, where

$$
H f(n)=\alpha f(n+1)+\beta f(n-1)
$$

is translation-invariant, and $V$ is a bounded potential. They treat a wider class of models than Goldsheid and Khoruzhenko, but take a different perspective. That is, they do not require that $\alpha \beta>0$, nor that the values $v_{r}$ of $V$ be chosen randomly. They assume instead that the values $v_{r}$ all lie in a given compact subset $S$ of $\mathbb{R}$, and that the potential satisfies an ergodicity property, which is indeed valid if the potential is chosen randomly as described above.

There are several ways of looking at the relationship between the two classes of results, which we henceforth call the HN and D models. The D model is in one sense what one obtains by combining all probability laws such that $v_{r} \in S$ for all $r$ into a single operator, so one would expect that its spectrum would be the union of the limiting spectra of all HN models. A partial proof of this is given below. From this point of view, the results of Goldsheid and Khoruzhenko are sharper, while the D model provides generic bounds on the asymptotic spectra of HN models for which the sharper results have not yet been computed, or are beyond the methods of those papers because, for example, $\alpha \beta<0$. In a separate paper [22], Martínez studies the D model, and obtains considerable improvements on the bounds given in $[9,10]$.

We next prove that the limit set $C$ of Goldsheid and Khoruzhenko is always contained in $\operatorname{Spec}\left(A_{\infty}\right)$.

THEOREM 4.1. If $v_{n} \in S$ for all $n$, then

$$
C \subseteq \operatorname{Spec}\left(A_{\infty}\right)
$$

Proof. Since $\operatorname{Spec}\left(A_{\infty}\right)$ is closed, it is sufficient to prove that

$$
\operatorname{Spec}(A) \subseteq \operatorname{Spec}\left(A_{\infty}\right)
$$

for all $M \times M$ matrices $A$ of the stated type. Given $A$, let $\tilde{A}$ denote its natural extension to a doubly infinite matrix with period $M$. If $\lambda$ is an eigenvalue of $A$, then there exists a bounded sequence $\{\phi(m)\}_{m \in \mathbb{Z}}$ with period $M$ such that

$$
\alpha \phi(m+1)+v_{m} \phi(m)+\beta \phi(m-1)=\lambda \phi(m)
$$

for all $m \in \mathbb{Z}$, where the random sequence $v_{1}, \ldots, v_{M}$ is extended periodically to $\mathbb{Z}$.

We claim that if $\varepsilon>0$ and $\phi_{\varepsilon}(m)=\mathrm{e}^{-\varepsilon|m|} \phi(m)$ for all $m \in \mathbb{Z}$, then $\phi \in l^{2}(\mathbb{Z})$ and

$$
\lim _{\varepsilon \rightarrow 0} \frac{\left\|\tilde{A} \phi_{\varepsilon}-\lambda \phi_{\varepsilon}\right\|}{\left\|\phi_{\varepsilon}\right\|}=0 .
$$

The method of proof is standard, but we include the details for completeness. By applying 
a space translation, we may assume that $\phi(0) \neq 0$. We then have

$$
\begin{aligned}
\left\|\phi_{\varepsilon}\right\|^{2} & =\sum_{m=-\infty}^{\infty} \mathrm{e}^{-2 \varepsilon|m|}|\phi(m)|^{2} \\
& \geqslant|\phi(0)|^{2} \sum_{r=0}^{\infty} \mathrm{e}^{-2 \varepsilon n r} \\
& \geqslant k_{1} \varepsilon^{-1},
\end{aligned}
$$

where $k_{1}>0$.

By using (18), we find that

$$
\begin{aligned}
\left(\tilde{A} \phi_{\varepsilon}-\lambda \phi_{\varepsilon}\right)(m)= & \alpha\left(\phi_{\varepsilon}(m+1)-\mathrm{e}^{-\varepsilon|m|} \phi(m+1)\right)+v_{m}\left(\phi_{\varepsilon}(m)-\mathrm{e}^{-\varepsilon|m|} \phi(m)\right) \\
& +\beta\left(\phi_{\varepsilon}(m-1)-\mathrm{e}^{-\varepsilon|m|} \phi(m-1)\right) \\
= & \alpha \phi(m+1)\left\{\mathrm{e}^{-\varepsilon|m+1|}-\mathrm{e}^{-\varepsilon|m|}\right\}+\beta \phi(m-1)\left\{\mathrm{e}^{-\varepsilon|m-1|}-\mathrm{e}^{-\varepsilon|m|}\right\} .
\end{aligned}
$$

Therefore,

$$
\left|\left(\tilde{A} \phi_{\varepsilon}-\lambda \phi_{\varepsilon}\right)(m)\right| \leqslant|\alpha|\|\phi\|_{\infty} \varepsilon \mathrm{e}^{-\varepsilon \min \{|m|,|m+1|\}}+|\beta|\|\phi\|_{\infty} \varepsilon \mathrm{e}^{-\varepsilon \min \{|m|,|m-1|\}} .
$$

This implies that

$$
\left\|\tilde{A} \phi_{\varepsilon}-\lambda \phi_{\varepsilon}\right\|^{2} \leqslant k_{2} \varepsilon
$$

We finally deduce (19) by combining (20) with (21).

Since $\varepsilon>0$ is arbitrary, this implies that $\lambda$ lies in $\operatorname{Spec}(\tilde{A})$. The inclusion

$$
\operatorname{Spec}(\tilde{A}) \subseteq \operatorname{Spec}\left(A_{\infty}\right)
$$

is proved by applying [10, Theorem 1].

\section{An abstract spectral bound}

We start by describing a method of obtaining a simultaneous bound, or enclosure, on the spectra of a family of related NSA matrices, without requiring any serious numerical computations. The bound is not as sharp as that of Section 3, but it remains valid in the limit as the sizes of the matrices increase to infinity. The main result of the section is Theorem 5.5, in which we apply the abstract ideas to the Anderson model in two space dimensions. The one-dimensional case has special features, which are treated by Martínez [22], who is able to give a more complete analysis of that case.

Our starting point is an abstract theorem.

THEOREM 5.1. Let $A=C+i S+V$, where $C, S$ and $V$ are bounded, self-adjoint operators on $\mathscr{H}$. Suppose that there exist a real constant $c$ and non-negative constants $\lambda, \mu$, and $\gamma_{c}$ such that

$$
S^{2}+\lambda C^{2} \leqslant \mu I \quad, \quad \gamma_{c}=\operatorname{dist}(c, \operatorname{Spec}(V)) .
$$

Then $x+i y \in \operatorname{Spec}(A)$ implies that

$$
y^{2} \leqslant(x-c)^{2}+\mu-\frac{\lambda \gamma_{c}^{2}}{1+\lambda} .
$$


Proof. On replacing $A$ by $A-c I$ (that is, $V$ by $V-c I$ ) it is easy to see that we need only consider the case $c=0$. The assumptions imply that

$$
(x+i y)^{2} \in \operatorname{Spec}\left(A^{2}\right) \subseteq \operatorname{Num}\left(A^{2}\right) .
$$

Therefore $x^{2}-y^{2} \geqslant v$, where $v$ is the bottom of the spectrum of $K=\left(A^{2}+A^{* 2}\right) / 2$. A direct computation shows that

$$
\begin{aligned}
K & =(C+V)^{2}-S^{2} \\
& \geqslant C^{2}+(C V+V C)+V^{2}+\lambda C^{2}-\mu I \\
& =\left((1+\lambda)^{1 / 2} C+(1+\lambda)^{-1 / 2} V\right)^{2}+\frac{\lambda}{1+\lambda} V^{2}-\mu I \\
& \geqslant \frac{\lambda}{1+\lambda} V^{2}-\mu I \\
& \geqslant\left(\frac{\lambda \gamma_{0}^{2}}{1+\lambda}-\mu\right) I .
\end{aligned}
$$

The statement of the theorem follows immediately.

We will apply the above theorem to $D$-dimensional operators of the form

$$
A=\sum_{m=1}^{D} a_{m} E_{m}+\sum_{m=1}^{D} b_{m} E_{m}^{*}+V
$$

where $\left\{E_{m}, E_{n}^{*}\right\}$ is a commuting family of unitary operators and $a_{m}$, and $b_{m}$ are real-valued coefficients. We may rewrite this in the form $A=C+i S+V$ by putting

$$
C=\sum_{m=1}^{D} \alpha_{m} C_{m} \quad \text { and } \quad S=\sum_{m=1}^{D} \beta_{m} S_{m}
$$

where $E_{m}=C_{m}+i S_{m}, \alpha_{m}=a_{m}+b_{m}$ and $\beta_{m}=a_{m}-b_{m}$.

LEMMA 5.2. Under the assumption (24), we have

$$
S^{2}+\lambda C^{2} \leqslant \mu I
$$

for all $\lambda \geqslant 0$, where

$$
\mu=\max \left\{\left(\sum_{m} \beta_{m} \sin \left(\theta_{m}\right)\right)^{2}+\lambda\left(\sum_{m} \alpha_{m} \cos \left(\theta_{m}\right)\right)^{2}: \theta \in[0,2 \pi]^{D}\right\}
$$

is a convex increasing function of $\lambda$ on $[0, \infty)$

Proof. We carry out the necessary calculations within the Gelfand representation of the commutative $\mathrm{C}^{*}$-algebra $\mathcal{A}$ generated by $\left\{E_{m}, E_{n}^{*}\right\}$. The maximal ideal space of $\mathcal{A}$ is homeomorphic to a compact subset $\Omega$ of $[0,2 \pi]^{D}$, and the operator $E_{m}$ is represented by the function $\left\{\theta \rightarrow \mathrm{e}^{i \theta_{m}}\right\} \in C(\Omega)$. The lemma follows by taking the supremum over all $\theta \in \Omega$ of the identity

$$
\left(S^{2}+\lambda C^{2}\right)(\theta)=\left(\sum_{m} \beta_{m} \sin \left(\theta_{m}\right)\right)^{2}+\lambda\left(\sum_{m} \alpha_{m} \cos \left(\theta_{m}\right)\right)^{2} .
$$

The next two lemmas provide weaker, but simpler, bounds than Lemma 5.2. Neither is better than the other for all values of the parameters, but if $\alpha_{m}=\alpha$ and $\beta_{m}=\beta$ for all $m$, 
then they both yield

$$
\mu=D^{2} \max \left\{\beta^{2}, \lambda \alpha^{2}\right\} .
$$

If, in addition, we put $\lambda=\beta^{2} / \alpha^{2}$, then all three lemmas yield

$$
\mu=D^{2} \beta^{2} \text {. }
$$

LEMma 5.3. Under the assumption (24), we have

$$
S^{2}+\lambda C^{2} \leqslant \mu I
$$

for all $\lambda \geqslant 0$, where

$$
\mu=D \max \left\{\left(\sum_{m} \beta_{m}^{2}\right), \lambda\left(\sum_{m} \alpha_{m}^{2}\right)\right\} .
$$

Proof. We have

$$
\begin{aligned}
S^{2}+\lambda C^{2} & =\left(\sum_{m} \beta_{m} S_{m}\right)^{2}+\lambda\left(\sum_{m} \alpha_{m} C_{m}\right)^{2} \\
& \leqslant\left(\sum_{m} \beta_{m}^{2}\right)\left(\sum_{m} S_{m}^{2}\right)+\lambda\left(\sum_{m} \alpha_{m}^{2}\right)\left(\sum_{m} C_{m}^{2}\right) \\
& \leqslant(\mu / D) \sum_{m}\left(S_{m}^{2}+C_{m}^{2}\right) \\
& =\mu .
\end{aligned}
$$

LEMMA 5.4. Under the assumption (24), we have

$$
S^{2}+\lambda C^{2} \leqslant \mu I
$$

for any $\lambda \geqslant 0$, where

$$
\mu=\left(\sum_{m} \delta_{m}\right)^{2} \quad \text { and } \quad \delta_{m}=\max \left\{\left|\beta_{m}\right|, \lambda^{1 / 2}\left|\alpha_{m}\right|\right\} .
$$

Proof. We have

$$
\begin{aligned}
S^{2}+\lambda C^{2} & \leqslant \sum_{m, n}\left(\left|\beta_{m} \beta_{n} S_{m} S_{n}\right|+\lambda\left|\alpha_{m} \alpha_{n} C_{m} C_{n}\right|\right) \\
& \leqslant \sum_{m, n} \delta_{m} \delta_{n}\left(\left|S_{m} S_{n}\right|+\left|C_{m} C_{n}\right|\right) .
\end{aligned}
$$

The proof is completed by using the fact that

$$
\left|S_{m} S_{n}\right|+\left|C_{m} C_{n}\right| \leqslant\left(S_{m}^{2}+C_{m}^{2}\right)^{1 / 2}\left(S_{n}^{2}+C_{n}^{2}\right)^{1 / 2} \leqslant 1
$$

Note that for the remainder of the paper, we assume that $A_{\infty}$ and $A$ are defined by (23) and (24), and act in $l^{2}\left(\mathbb{Z}^{D}\right)$ and $l^{2}\left(\{1, \ldots, M\}^{D}\right)$, respectively, subject to periodic boundary conditions. We assume that the unitary operators $E_{m}$ are given by $\left(E_{m} f\right)(x)=f\left(x+e_{m}\right)$ for all $x \in \mathbb{Z}^{D}$ and by $x \in\{1, \ldots, M\}^{D}$, respectively, where $\left(e_{m}\right)_{n}=\delta_{m, n}$. It is obvious that the family of all such operators commutes. We assume that the bounded operator $V$ is of the form $(V f)(x)=V(x) f(x)$. The constants $\gamma_{c}$ of (22) are given by

$$
\gamma_{c}=\inf \left\{|V(x)-c|: x \in \mathbb{Z}^{D}\right\},
$$

with the obvious modification for $\{1, \ldots, M\}^{D}$. 
A wide variety of such examples can be studied, but for the sake of definiteness we concentrate on the two-dimensional case and suppose that the constants in (23) satisfy $a_{1}=a_{2}=a$ and $b_{1}=b_{2}=b$. We put $\alpha=a+b$ and $\beta=a-b$. We also assume that $V(x)= \pm \gamma$ for every $x$ in $\mathbb{Z}^{2}$ (or in $\{1, \ldots, M\}^{2}$ ).

THEOREM 5.5. If $\gamma=0$, then $\operatorname{Spec}\left(A_{\infty}\right)$ is the region $E$ inside or on the ellipse with centre 0 and vertices $( \pm 2 \alpha, 0)$ and $(0, \pm 2 \beta)$. Also, $\operatorname{Spec}(A) \subset E$ and $\operatorname{Spec}(A) \rightarrow E$ as $M \rightarrow \infty$. If $\gamma>0$ and $A_{\infty}$ is pseudo-ergodic in the sense of [10], then

$$
\operatorname{Spec}\left(A_{\infty}\right) \supseteq(E+\gamma) \cup(E-\gamma) .
$$

Moreover,

$$
\operatorname{Spec}(A) \subseteq \operatorname{Spec}\left(A_{\infty}\right) \subseteq T:=\left\{x+i y: y^{2} \leqslant \tau(x)\right\}
$$

where

$$
\tau(x)=\inf _{c \in \mathbb{R}}\left\{(x-c)^{2}+4 \beta^{2}-\frac{\beta^{2} \gamma_{c}^{2}}{\alpha^{2}+\beta^{2}}\right\}
$$

and

$$
\gamma_{c}=\min \{|\gamma-c|,|\gamma+c|\}
$$

Proof. The first statement is a routine exercise using Fourier analysis. The others follow by the methods of this paper. We put $\lambda=\beta^{2} / \alpha^{2}$ and obtain $\mu=4 \beta^{2}$ from (27). We then apply Theorem 5.1 to derive (29), (30) and (31).

Corollary 5.6. If $y \in \mathbb{R}$ and $i y \in \operatorname{Spec}\left(A_{\infty}\right)$, then

$$
y^{2} \leqslant \tau(0)=4 \beta^{2}-\frac{\beta^{2} \gamma^{2}}{\alpha^{2}+\beta^{2}} .
$$

No such y exists if $\gamma^{2}>4\left(\alpha^{2}+\beta^{2}\right)$.

The above corollary is sharp if $\alpha=0$, but in other cases we can prove the following lemma. Much more can be proved by carrying out a complete Bloch-type analysis of $A$ for various periodic potentials $V$ with values in $\{-\gamma, \gamma\}$. See Example 6.8 for an illustration of this lemma.

LemMA 5.7. If $y=\sqrt{4 \beta^{2}-\gamma^{2}}>0$, then $\pm i y \in \operatorname{Spec}\left(A_{\infty}\right)$.

Proof. It is sufficient to construct a bounded periodic eigenfunction $f$ with eigenvalue $\pm i y$ for an infinite periodic matrix $A$ of the stated form, where

$$
V(m, n)=(-1)^{m+n} \gamma \text {. }
$$

This is achieved by taking $f$ to be the periodic extension to $\mathbb{Z}^{2}$ of the function given on $\{1, \ldots, 4\}^{2}$ by

$$
f(m, n)=\left(\begin{array}{rrrr}
u & v & -u & -v \\
v & -u & -v & u \\
-u & -v & u & v \\
-v & u & v & -u
\end{array}\right)
$$

for some constants $u, v$. This leads one to a two-dimensional eigenvalue problem with the stated solutions. 


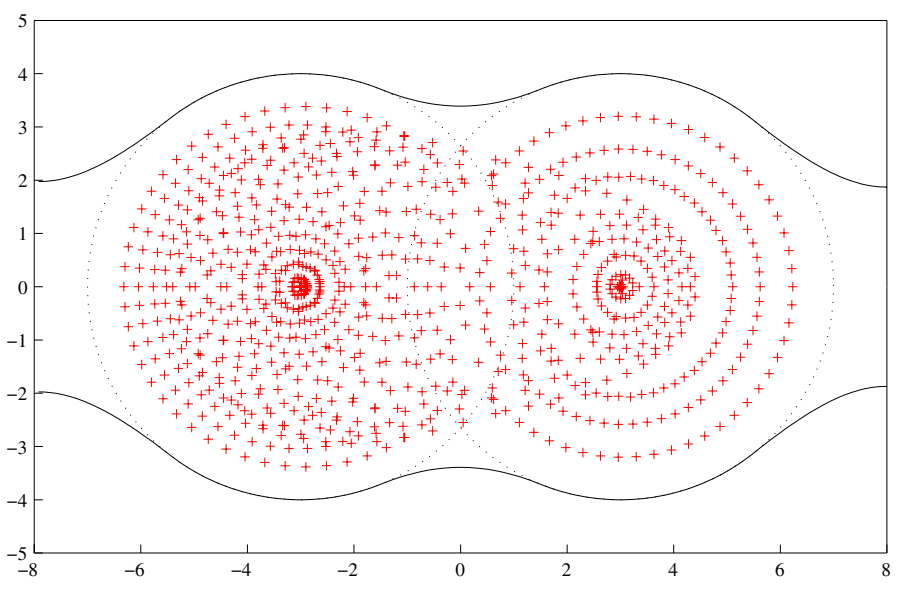

Figure 3: Spectrum of the NSA operator of Example 6.1.

\section{Applications}

We finally compare the above theoretical results with numerical computations. All of the examples below are set on two-dimensional $M \times M$ lattices with periodic boundary conditions, so the matrices have sizes $M^{2} \times M^{2}$. The meanings of the parameters and the operator $A$ are specified in the paragraph just before Theorem 5.5. We show that the spectral properties of the $\mathrm{HN}$ model depend heavily on the probability law governing the values of the potential. At one extreme are potentials that are constant or periodic with period 2, and at the other are random potentials that take independent values at each point. Several of the figures are similar to those in $[7, \mathbf{1 8}, 19,23]$, but we compare the results with our rigorous bounds, and also describe some phenomena which are new, even at the numerical level.

If the values $\pm \gamma$ of the potential at neighbouring points are strongly correlated, then the potential is constant on large connected domains, and the spectral properties of the HN model should depend upon the domain boundaries. We show below that in such situations the spectrum of the D model is quite similar to the spectrum of the HN model, even for quite small periodic lattices: effects that are extremely improbable when the values of the potential at different sites are independent become the dominant terms for highly correlated potentials. We also show (numerically) that the eigenfunctions are strongly localized, and that their maxima are concentrated on the domain boundaries. These effects can be demonstrated quite easily in one dimension, where the domain boundaries are isolated points, but we show that they persist in the more physically realistic two-dimensional case.

EXAMPLE 6.1. Figure 3 shows the eigenvalues of an operator $A$ of the type described above, with $M=30, a_{1}=a_{2}=2$, and $b_{1}=b_{2}=0$. These choices yield $\alpha=\beta=2$, so $E$ is a circular ball with centre 0 and radius 4 . The potential $V$ is defined by

$$
V(m, n)=\left\{\begin{aligned}
\gamma & \text { if }(m-M / 2)^{2}+(n-M / 2)^{2} \geqslant M^{2} / 5, \\
-\gamma & \text { otherwise, }
\end{aligned}\right.
$$

where $\gamma=3$. The figure also shows the balls with centres $\pm \gamma$ and radius 4 related to (28) and curves $y= \pm \tau(x)^{1 / 2}$ related to (29). We computed $\tau(x)$ numerically using (30). Information about the eigenfunctions of this example may be found in Example 6.5. 


\section{Spectral bounds}

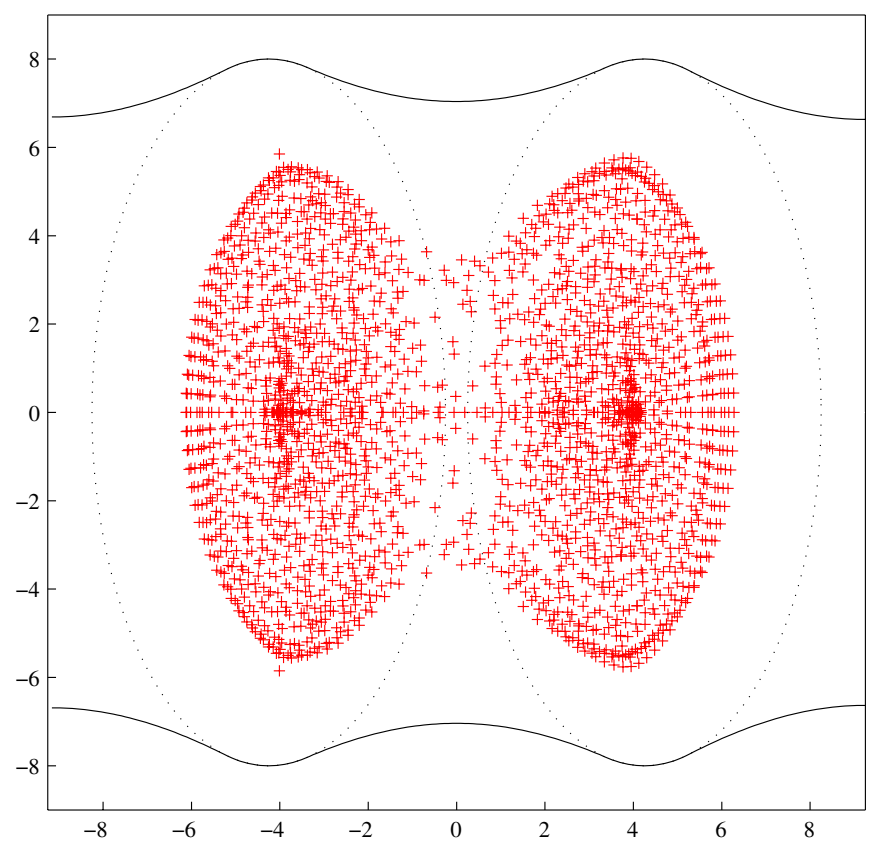

Figure 4: Spectrum of the NSA operator of Example 6.2.

It follows from [10, Theorems 3 and 4] and Theorem 5.5 that

$$
\operatorname{Spec}(A) \subseteq \operatorname{Spec}\left(A_{\infty}\right)=(E+\gamma) \cup(E-\gamma) .
$$

We conjecture that $\operatorname{Spec}(A)$ converges to $\operatorname{Spec}\left(A_{\infty}\right)$ as $M \rightarrow \infty$. This is not easy to demonstrate numerically, let alone prove, because the difficulty of computing the eigenvalues of $A$ increases rapidly with $M$. In Table 1 , we list the largest real eigenvalue $\lambda_{M}$ of $A$ as a function of $M$, computed using Matlab's 'eigs' routine. The data provide limited support for the conjecture that $\lambda_{M} \rightarrow \gamma+2 \beta=7$ as $M \rightarrow \infty$. One must, however, be cautious about accepting the results of eigenvalue calculations for large, highly non-self-adjoint matrices, because of problems with ill-conditioning (that is, pseudospectra). It follows from (33) that there are no eigenvalues of $A$ in the curvilinear triangles of Figure 3.

EXAmPLE 6.2. Figure 4 shows the eigenvalues of a similar operator, but with $M=48$, $a_{1}=a_{2}=3$ and $b_{1}=b_{2}=-1$. These choices yield $\alpha=2$ and $\beta=4$. The potential $V$

Table 1: Dependence of the largest eigenvalue $\lambda_{M}$ of $A$ on $M$.

\begin{tabular}{rc}
\hline$M$ & $\lambda_{M}$ \\
\hline 25 & 6.176 \\
50 & 6.420 \\
100 & 6.589 \\
150 & 6.657 \\
200 & 6.691 \\
250 & 6.713 \\
\hline
\end{tabular}




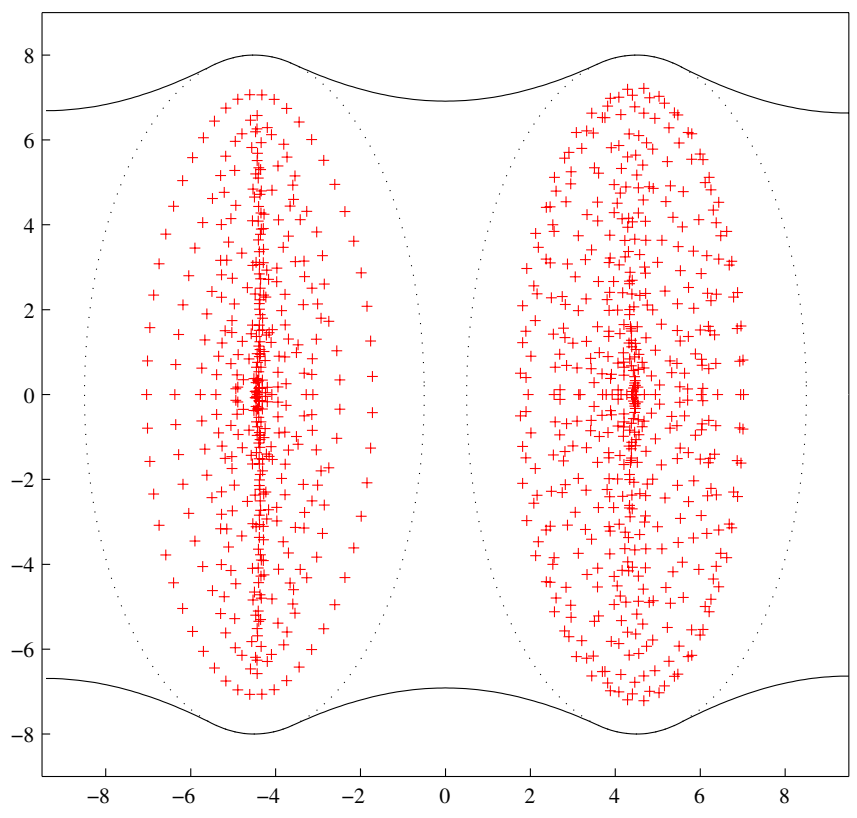

Figure 5: Spectrum of the NSA operator of Example 6.3.

takes the values $\pm \gamma$ with equal probabilities, independently at each site, with $\gamma=4.5$. It may be seen that there are several eigenvalues outside the two ellipses but between the curves $y= \pm \tau(x)$. If one compares this figure with the corresponding one for $M=24$, one finds that the envelope of the eigenvalues is almost the same, but for $M=48$ they are more closely packed. We conjecture that $\operatorname{Spec}(A)$ converges as $M \rightarrow \infty$ to a proper subset of the set $T$ defined in Theorem 5.5.

EXAMPLE 6.3. We again consider the case $M=30, a_{1}=a_{2}=3, b_{1}=b_{2}=-1$, so that $\alpha=2$ and $\beta=4$. The potential $V$ takes the values $\pm \gamma$ with $\gamma=4.5$, but we make the choices by a new procedure, motivated by the wish to have several irregular regions of intermediate size within which $V(x, y)$ has constant sign. We achieve this rather crudely by dividing $\{1, \ldots, M\}^{2}$ into squares of size $Q \times Q$, where $Q=6$ is a factor of $M$. We take $V$ to be constant within each square, but choose its sign independently in different squares. Figure 5 shows that the distribution of the eigenvalues is quite different from that in Example 6.2, and that it conforms much more closely to the two elliptical regions. It appears that as $Q$ increases, the spectrum converges to the union of the two elliptical regions, provided that the size of $M$ grows correspondingly. The particular choice of signs that leads to Figure 5 is as follows.

$$
\begin{array}{ccccc}
+ & + & + & + & + \\
- & + & + & - & - \\
+ & - & - & - & + \\
+ & + & - & - & + \\
+ & - & + & - & -
\end{array}
$$




\section{Spectral bounds}

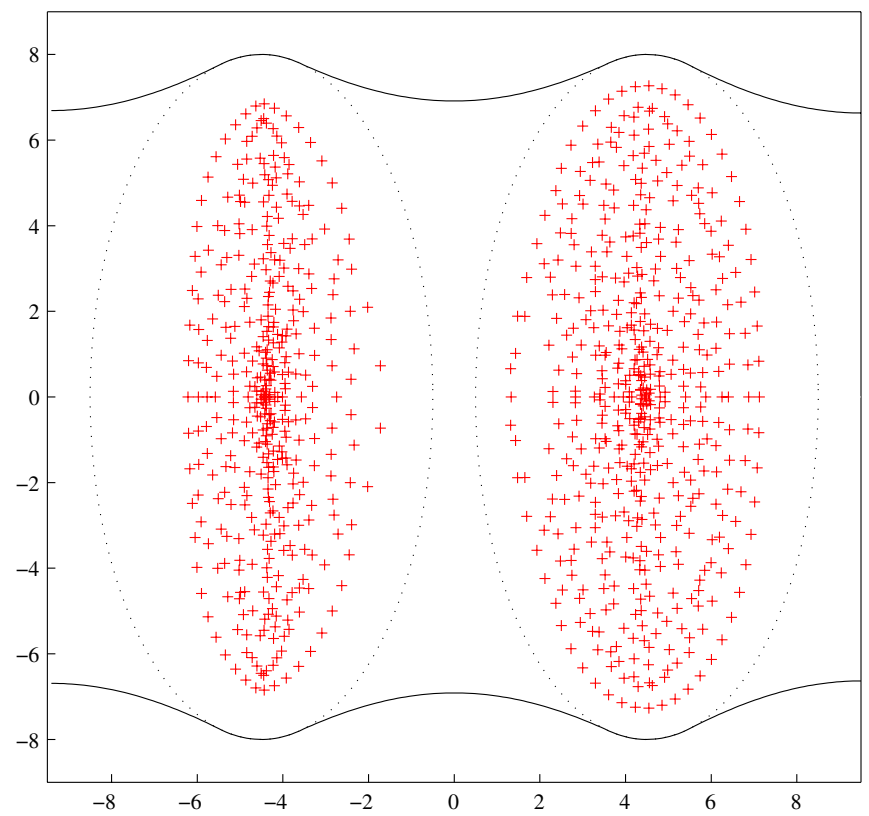

Figure 6: Spectrum of the NSA operator of Example 6.4.

EXAmPLE 6.4. We consider the case $M=30, a_{1}=a_{2}=3, b_{1}=b_{2}=-1$, so that $\alpha=2$ and $\beta=4$. The potential $V$ again takes the values $\pm \gamma$ with $\gamma=4.5$, but we arrange that there is a high correlation between the values of $V$ at nearby points by using a cellular automaton $\mathcal{F}$ to generate $V$. We put $V=\gamma \mathcal{F}^{5}(W)$, where $W(m, n)= \pm 1$ for all $m, n$, the sign being chosen independently and randomly at each site. For any configuration $X:\{1, \ldots, M\}^{2} \rightarrow\{-1,1\}$, we define $Y=\mathcal{F}(X)$ by

$$
Y(m, n)=\left\{\begin{aligned}
1 & \text { if } \sum_{(r, s) \in S_{(m, n)}} X(r, s)>0, \\
-1 & \text { otherwise, }
\end{aligned}\right.
$$

where

$$
S_{(m, n)}=\{(r, s):|r-m| \leqslant 1 \text { and }|s-n| \leqslant 1\},
$$

and we take into account the periodicity of the square $\{1, \ldots, M\}^{2}$.

Figure 6 shows that the distribution of the eigenvalues is quite different from that in Example 6.2, and that it conforms much more closely to the two elliptical regions.

We demonstrate numerically another feature of the same example: that the eigenfunctions of the operator $A$ are strongly localized. Let $\phi: S \longrightarrow \mathbb{C}$ be any eigenfunction of $A$, where $S=\{1, \ldots, M\}^{2}$, and suppose that $\|\phi\|_{2}=1$, so that $\rho(x, y)=|\phi(x, y)|^{2}$ is a probability distribution on $S$. If $\rho$ were approximately constant, then one would have $\|\rho\|_{\infty} \sim 0.001$. We computed $\|\rho\|_{\infty}$ for each eigenfunction, and determined from the data that 524 out of the 900 eigenfunctions of $A$ have $\|\rho\|_{\infty}>0.1$, while 830 have $\|\rho\|_{\infty}>0.04$. 


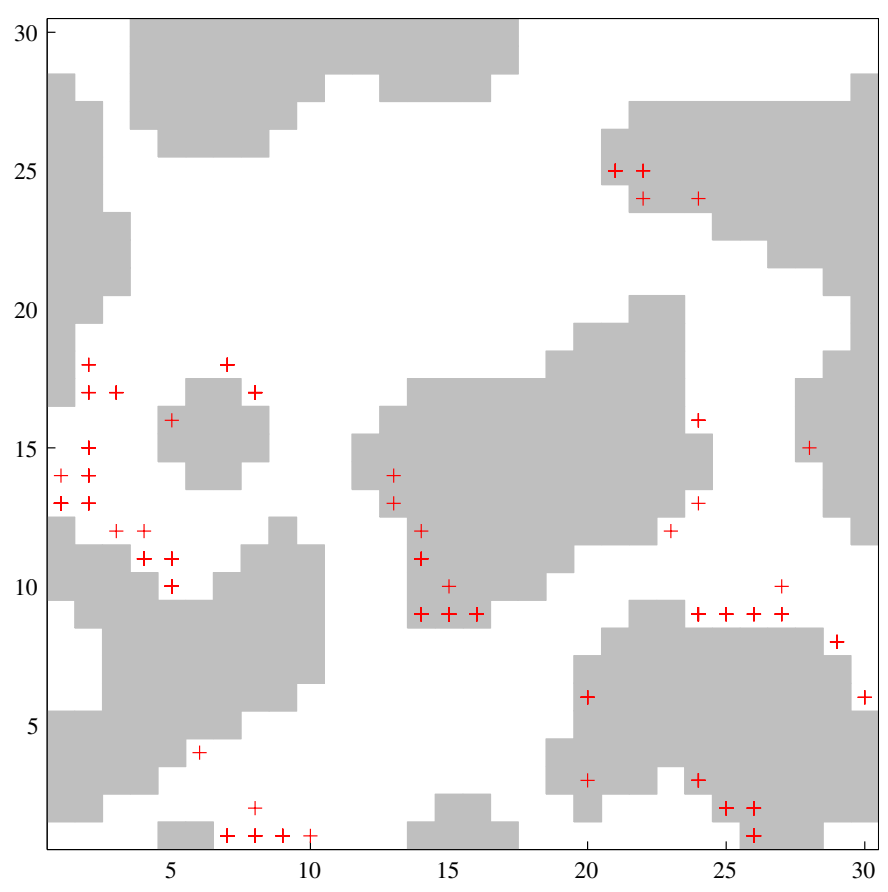

Figure 7: Distribution of the maxima of the eigenfunctions of Example 6.4.

Given the above, it is natural to examine the distribution of the maxima of the eigenfunctions. We determined the maximizing point $(m, n) \in S$ for each function $\rho$, and plotted these using ' + ' in Figure 7. The gray region in this figure is the union of unit squares centred at the points $(m, n)$ where $V(m, n)=-\gamma$, the corresponding region for $V(m, n)=\gamma$ being white. It may be seen that all of the eigenfunctions take their maximum values at or very near the domain boundaries. Taking into account the periodic boundary conditions, we see that there is one region throughout which $V(m, n)=\gamma$ and four regions in which $V(m, n)=-\gamma$.

EXAMPLE 6.5. Other choices of the parameters in Example 6.4 yield similar results, provided that the operator is non-self-adjoint. Whether or not the potential is produced randomly is not relevant; for example, Example 6.1 leads to Figure 8. It is worth noting that the maxima of the eigenfunctions are concentrated on the part of the boundary whose normals are roughly parallel to the vector $(1,1)$. The same effect occurs in Example 6.4, but less clearly. This might be 'explained' by considering the operator $A$ to be a discrete approximation to an operator of the form

$$
(H f)(x, y)=\frac{\partial f}{\partial x}+\frac{\partial f}{\partial y}+V(x, y) f(x, y)+c f(x, y)
$$

acting in $L^{2}\left((0,1)^{2}\right)$, subject to periodic boundary conditions. The operator $H$ is associated with a flow which has orbits parallel to the direction of $(1,1)$. 


\section{Spectral bounds}

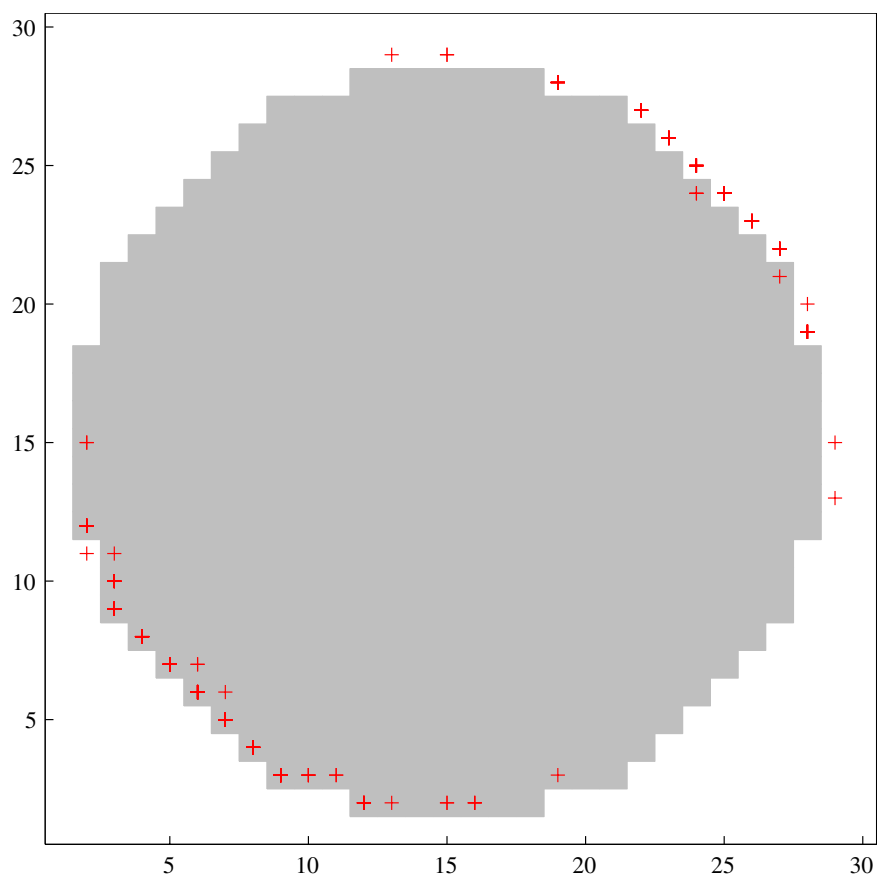

Figure 8: Distribution of the maxima of the eigenfunctions of Example 6.1.

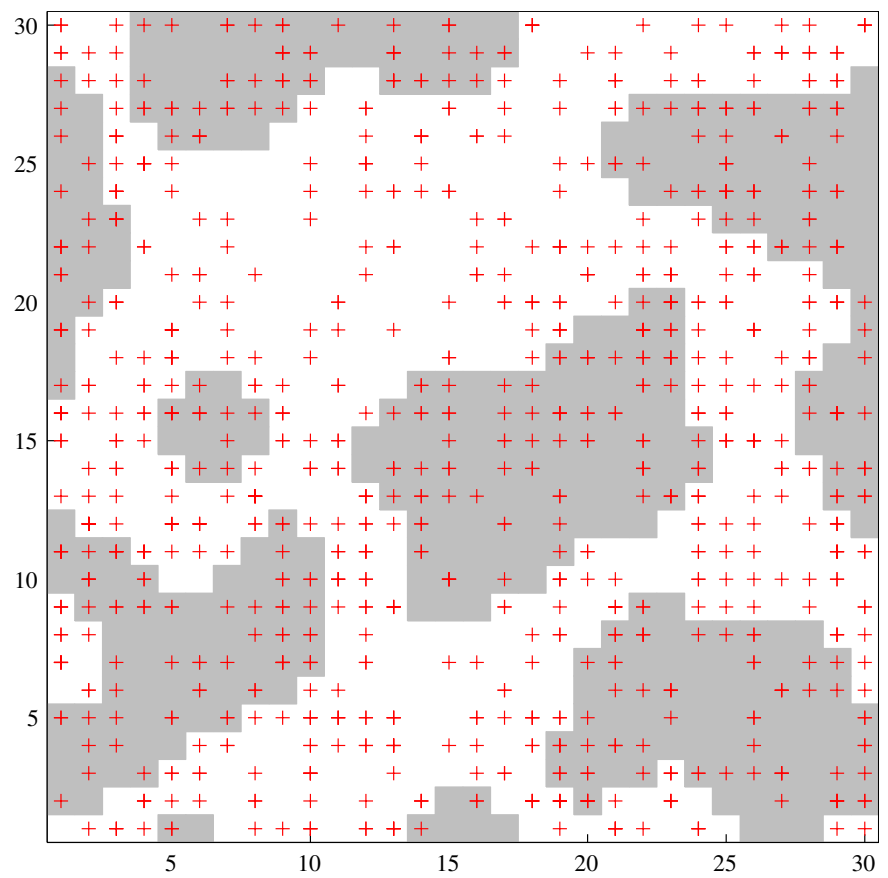

Figure 9: Distribution of the maxima of the eigenfunctions of Example 6.6. 


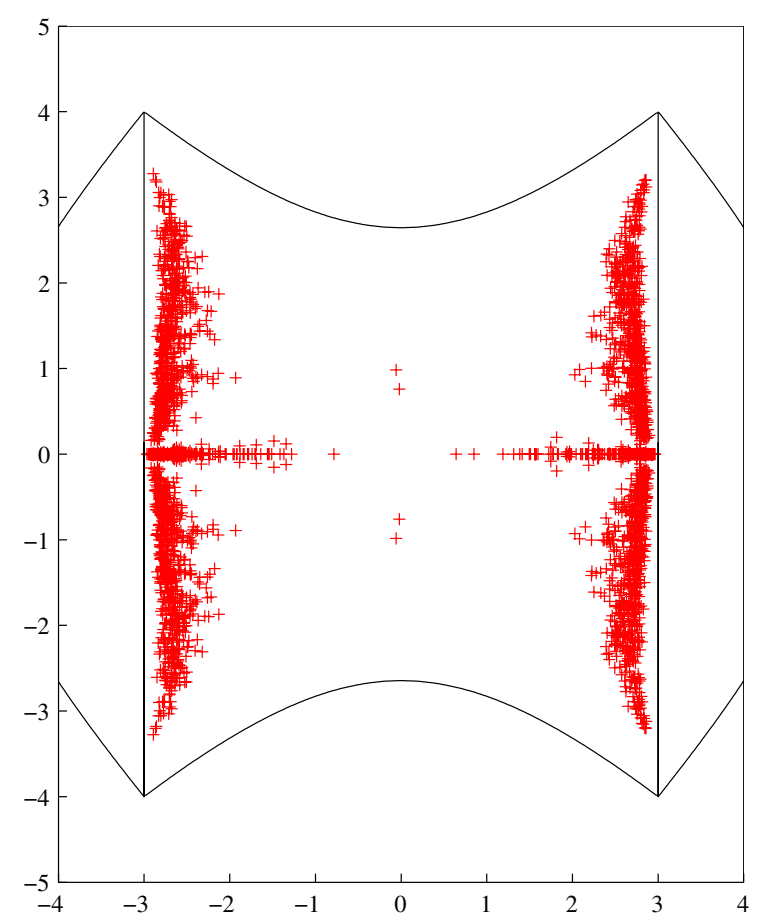

Figure 10: Spectrum of the NSA operator of Example 6.7.

EXAMPLE 6.6. We considered a self-adjoint example for comparison. We put $M=30$, $a_{1}=a_{2}=b_{1}=b_{2}=1$, so that $\alpha=2$ and $\beta=0$, and chose the same potential $V$ as in Example 6.4. The phenomena described in Example 6.4 no longer occurred. Only 49 out of the 900 eigenfunctions of $A$ have $\|\rho\|_{\infty}>0.1$. Figure 9 was produced in the same way as Figure 7, but the maxima are scattered over the whole of the square, with no tendency to concentrate on the domain boundaries.

We obtained similar results on putting $M=50$ in this example.

EXAmPLE 6.7. Figure 10 shows the eigenvalues of the operator $A$ with $M=50, a_{1}=$ $a_{2}=1, b_{1}=b_{2}=-1$. These choices yield $\alpha=0$ and $\beta=2$. The potential $V$ takes the values $\pm \gamma$ with equal probabilities, independently at each site, where $\gamma=3$. The set $(E+\gamma) \cup(E-\gamma)$ is represented by the two vertical straight lines in Figure 10, and we see that none of the eigenvalues lies in the set. All of the eigenvalues lie between the two solid curves, which are approximations to $y= \pm \tau(x)^{1 / 2}$. The approximation is much better within the range $-\gamma \leqslant x \leqslant \gamma$ than it is outside this interval, for reasons explained in Lemma 3.6 and Example 3.7.

The four points close to the $y$-axis are absent in the corresponding figure for $M=30$, for which the points nearest to the origin are approximately \pm 0.9 . For this reason, we do not believe that the spectrum shown in Figure 10 is close to the limit of the spectrum as $M \rightarrow \infty$. 


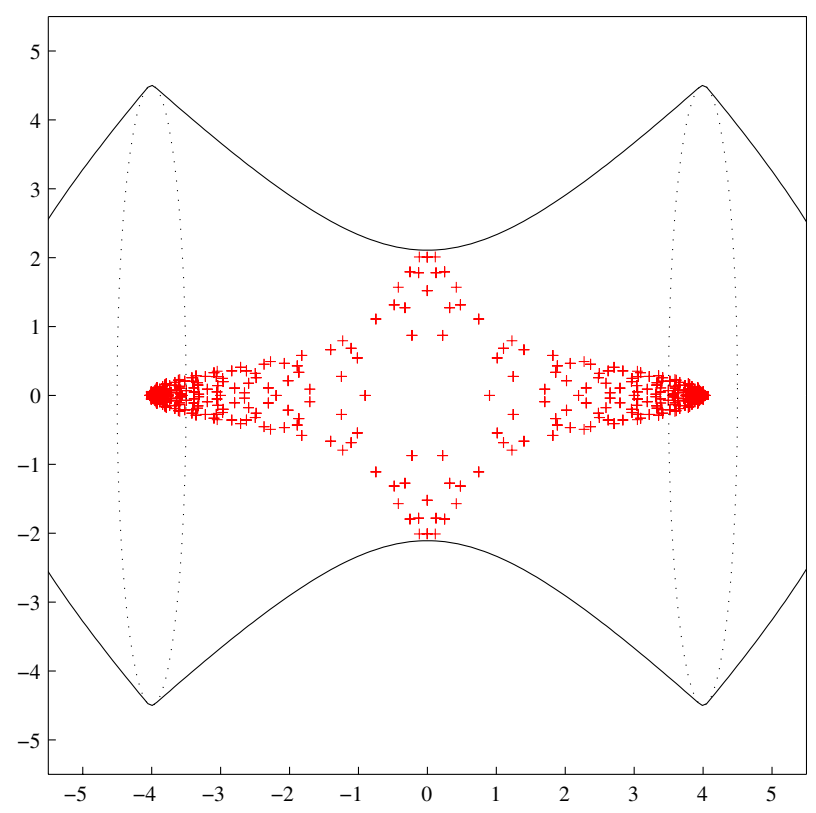

Figure 11: Spectrum of the NSA operator of Example 6.8.

Table 2 lists $n_{M}$ and $\lambda_{M}$ against $M$, where $n_{M}$ is the number of eigenvalues of $A$ satisfying $-0.1<x<0.1$ and $\lambda_{M}$ is the smallest real eigenvalue. Both were computed using Matlab's 'eigs' function. Since the matrices $A$ are particular choices out of a random ensemble, the results can only be regarded as indicative. They are consistent with the conjecture that the number of eigenvalues close to the imaginary axis increases indefinitely with $M$.

The one-dimensional version of this example was studied in considerable detail by Martínez [22]. She gave a theoretical explanation of the detailed structure of the spectrum seen in numerical studies. In the two-dimensional case, the structure is much less clear, presumably because the variety of local patterns of the values of $V(m, n)$ is much greater.

EXAMPLE 6.8. Figure 11 illustrates the conclusion of Lemma 5.7. It shows the eigenvalues of the operator $A$ with $M=30, a_{1}=a_{2}=1.25, b_{1}=b_{2}=-1$. These choices yield $\alpha=0.25$ and $\beta=2.25$. The potential $V$ is given by $V(m, n)=(-1)^{m+n} \gamma$, where $\gamma=4$. We see that the largest purely imaginary eigenvalue is quite close to the curve $y=\tau(x)$.

Table 2: Some data concerning the eigenvalues of $A$.

\begin{tabular}{rrr}
\hline$M$ & $n_{M}$ & \multicolumn{1}{c}{$\lambda_{M}$} \\
\hline 50 & 6 & -0.1490 \\
100 & 6 & 0.0766 \\
150 & 12 & -0.2595 \\
200 & 14 & 0.0554 \\
\hline
\end{tabular}


Acknowledgements. I acknowledge valuable exchanges with H. G. Dales, I. Goldsheid,

A. Greenbaum, B. A. Khoruzhenko, L. N. Trefethen and, most of all, C. Martínez.

This work was supported by EPSRC grant number GR/R81756.

\section{References}

1. A. Abramov, A. Aslanyan and E. B. Davies, 'Bounds on complex eigenvalues and resonances', J. Phys. A Math. Gen. 34 (2001) 52-72. 24

2. B. M. Brown and M. S. P. EAstham, 'Analytic continuation and resonance free regions for Sturm-Liouville potentials with power decay', J. Comp. Appl. Math. 148 (2002) 49-63. 24

3. A. BöтtсHER, 'Pseudospectra and singular values of large convolution operators', J. Integral Equations Appl. 6 (1994) 267-301. 21

4. A. BötTCHer and B. Silbermann, Introduction to large truncated Toeplitz matrices (Springer, New York, 1998). 21

5. E. BRÉzIN and A. ZEE, 'Non-Hermitean delocalization, multiple scattering and bounds', Nuclear Phys. B 509 (1998) 599-614. 30

6. J. Burke and A. Greenbaum, 'Some equivalent characterizations of the polynomial numerical hull of degree $k$ ', Preprint, Nov. 2004;

http://web.comlab.ox.ac.uk/oucl/publications/natr/na-04-29.html. 18,19

7. H. A. Dahmen, D. R. Nelson and N. M. Shnerb, 'Population dynamics and non-hermitian localization', Statistical mechanics of biocomplexity, (ed. D. Reguera, J. M. G. Vilar and J. M. Rubi, Springer, Berlin 1999) 124-151. 30, 36

8. E. B. DAvies, One-parameter semigroups (Academic Press, London, 1980). 25

9. E. B. DAviES, 'Spectral properties of random non-self-adjoint matrices and operators', Proc. Roy. Soc. London A 457 (2001) 191-206. 30, 31

10. E. B. Davies, 'Spectral theory of pseudo-ergodic operators', Commun. Math. Phys. 216 (2001) 687-704. 30, 31, 32, 35, 37

11. E. B. DAviES and J. NATH, 'Schrödinger operators with slowly decaying potentials', J. Comp. Appl. Math. 148 (2002) 1-28. 24

12. CH. Davis and A. SAlEmi, 'On polynomial numerical hulls of normal matrices', Lin. Alg. Appl. 383 (2004) 151-161. 18

13. J. Feinberg and A. ZeE, 'Spectral curves of non-hermitian hamiltonians', Nucl. Phys. B 552 (1999) 599-623. 30

14. I. Ya. Goldsheid and B. A. Khoruzhenko, 'Distribution of eigenvalues in nonHermitian Anderson model', Phys. Rev. Lett. 80 (1998) 2897-2901. 30, 31

15. I. YA. GoldSheid and B. A. KHORUZHEnKo, 'Eigenvalue curves of asymmetric tridiagonal random matrices', Electronic J. Prob. 5 (2000) Paper 16, 1-28. 30, 31

16. I. YA. GoldsheID and B. A. KHORUZHEnKo, 'Regular spacings of complex eigenvalues in the one-dimensional non-Hermitian Anderson model', Comm. Math. Phys. 238 (2003) 505-524. 30, 31

17. A. Greenbaum, 'Generalizations of the field of values useful in the study of polynomial functions of a matrix’, Linear Algebra Appl. 347 (2002) 233-249. 18 
18. N. HatAno and D. R. Nelson, 'Vortex pinning and non-Hermitian quantum mechanics', Phys. Rev. B 56 (1997) 8651-8673. 30, 36

19. N. Hatano and D. R. Nelson, 'Non-Hermitian delocalization and eigenfunctions', Phys. Rev. B 58 (1998) 8384-8390. 30, 36

20. H. LANGer and C. Tretter, 'Spectral decomposition of some nonselfadjoint block operator matrices', J. Operator Theory 39 (1998) 339-359. 18

21. H. Langer, A. Markus, V. Matsaev and C Tretter, 'A new concept for block operator matrices. The quadratic numerical range', Linear Algebra Appl. 330 (2001) 89-112. 18

22. C. Martínez, 'The non-self-adjoint Anderson model', J. Oper. Theory, to appear. 17, 30, 31, 32, 43

23. D. R. Nelson and N. M. Shnerb, 'Non-Hermitian localization and population biology', Phys. Rev. E 58 (1998) 1383-1403. 30, 36

24. O. Nevanlinna, Convergence of iterations for linear equations (Birkhauser, Basel, 1993). 18, 19, 20

25. S. C. Reddy, P. J. Schmid and D. S. Henningson, 'Pseudospectra of the OrrSommerfeld operator', SIAM J. Appl. Math. 53 (1993) 15--47. 27

26. P. RedPARTH, 'Spectral properties of non-self-adjoint operators in the semi-classical regime', J. Differential Equations 177 (2001) 307-330. 27

27. A. ShKalikov, 'The limit behaviour of the spectrum for large parameter values in a model problem', Math. Notes 62 (1997) No. 6. 27

28. S. D. Stoller, W. Happer and F. J. Dyson, 'Transverse spin relaxation in inhomogeneous magnetic fields', Phys. Rev. A 44 (1991) 7459-7477. 27

29. L. N. Trefethen, 'Pseudospectra of matrices', Numerical Analysis 1991 (ed. D. F. Griffiths and G. A. Watson, Longman Sci. Tech. Publ., Harlow, UK, 1992) 234-266. 21

30. L. N. Trefethen and M. Embree, Spectra and pseudospectra (Princeton Univ. Press, to appear). $17,21,30$

31. L. N. Trefethen, M. Contedini and M. Embree, 'Spectra, pseudospectra, and localization for random bidiagonal matrices', Comm. Pure Appl. Math. 54 (2001) 595-623. 30

E. B. Davies E.Brian.Davies@kcl.ac.uk

http://www.mth.kcl.ac.uk/staff/eb_davies.html

Department of Mathematics

King's College

Strand

London WC2R 2LS

United Kingdom 